\title{
Asthma inhaler adherence determinants in adults: systematic review of observational data
}

\author{
Alexandra L. Dima ${ }^{1}$, Gimena Hernandez ${ }^{2,3}$, Oriol Cunillera ${ }^{2}$, \\ Montserrat Ferrer ${ }^{2,3,4}$, Marijn de Bruin ${ }^{1,5}$ and the ASTRO-LAB group
}

Affiliations: ${ }^{1}$ Dept of Communication Science, ASCoR, University of Amsterdam, Amsterdam, The Netherlands. ${ }^{2}$ Health Services Research Unit, IMIM (Hospital del Mar Research Institute), Barcelona, Spain. ${ }^{3}$ Dept of Paediatrics, Obstetrics and Gynaecology and Preventative Medicine, Universitat Autónoma de Barcelona, Barcelona, Spain. ${ }^{4}$ CIBER Epidemiología y Salud Pública (CIBERESP), Spain. ${ }^{5}$ Aberdeen Health Psychology Group, Institute of Applied Health Sciences, University of Aberdeen, Aberdeen, UK.

Correspondence: Alexandra L. Dima, Dept of Communication Science, ASCoR, University of Amsterdam, Nieuwe Achtergracht 1661018 WV, PO Box 15791, 1001NG Amsterdam, The Netherlands.

E-mail: a.dimađuva.nl

ABSTRACT Nonadherence to inhaled medication leads to poor asthma control and increased healthcare utilisation. Many studies exploring adherence determinants have been conducted, but summaries of the evidence are scarce. We performed a systematic review of observational research on determinants of asthma inhaler adherence among adults.

We searched for articles in English reporting quantitative observational studies on inhaler adherence correlates among adults in developed countries, published in EMBASE, Medline, PsychInfo and PsychArticles in 1990-2014. Two coders independently assessed eligibility and extracted data, and assessed study quality. Results were summarised qualitatively into social and economic, and healthcare-, therapy-, condition- and patient-related factors.

The 51 studies included mainly examined patient-related factors and found consistent links between adherence and stronger inhaler-necessity beliefs, and possibly older age. There was limited evidence on the relevance of other determinants, partly due to study heterogeneity regarding the types of determinants examined. Methodological quality varied considerably and studies performed generally poorly on their definitions of variables and measures, risk of bias, sample size and data analysis.

A broader adoption of common methodological standards and health behaviour theories is needed before cumulative science on the determinants of adherence to asthma inhalers among adults can develop further.

@ERSpublications

Major opportunities for strengthening evidence on determinants of nonadherence to asthma inhalers: methods and theory http://ow.ly/DY4vr

This article has been corrected according to the author correction published in the June 2016 issue of the European Respiratory Journal.

This article has supplementary material available from erj.ersjournals.com

Received: May 022014 | Accepted after revision: Oct 312014 | First published online: Dec 102014

Support statement: The research leading to these results has received funding from the European Community 7 th Framework Programme (FP7/2007-2013; grant agreement no. 282593). Funding information for this article has been deposited with FundRef.

Conflict of interest: Disclosures can be found alongside the online version of this article at erj.ersjournals.com

Copyright OERS 2015 


\section{Introduction}

The introduction of inhaled medication as the primary treatment for asthma has led to substantial improvements in asthma control [1,2]. However, uncontrolled asthma is still common and represents a considerable burden to patients and society [3,4]. An important reason for poor asthma control and, consequently, increased healthcare expenditure is suboptimal adherence to the prescribed regimen [5-7]. To date, few adherence interventions evaluated in asthma treatment have been found to be (cost-)effective [8-10]. A systematic review of observational evidence on adherence determinants could help identify the patients most at-risk for nonadherence and the key drivers of nonadherence that can be modified in adherence interventions.

Although several narrative reviews on determinants of adherence to asthma medication have been conducted [11-18], only two systematic reviews on observational research are available. Both examined adherence to inhaled corticosteroids (ICS): one focused on children [19], the other exclusively evaluated the role of illness and treatment perceptions in adults [20]. Neither examined the quality of the methodology of included studies, which is important in interpreting empirical evidence [21-23]. To our knowledge, no comprehensive systematic review of factors related to adherence to inhaled medication in adults with asthma has been published to date.

The objective of this study was to synthesise the current observational evidence on determinants of inhaler adherence in asthmatic adults through a systematic review, including a critical appraisal of the methodological quality of the studies, and develop recommendations for future research in this domain.

\section{Methods}

Literature search and study selection

EMBASE, Medline, PsychInfo and PsychArticles were searched for manuscripts published between January 1, 1990 and June 26, 2014 with keywords on asthma, adherence, persistence, compliance, concordance, determinant, cause, influence, barrier and facilitator (Supplementary material 1). Eligibility was determined using the following criteria: peer-reviewed article in English; reporting an empirical quantitative observational study (cross-sectional or longitudinal designs); presenting results on adult (aged $>18$ years) asthma patients living in developed countries [24]; investigating one or more predictor of adherence to inhaled asthma medication; and describing the adherence measurement procedure. The selection was initially based on the information in the title and abstract; if inconclusive, the entire manuscript was examined. Two reviewers (A.L. Dima and O. Cunillera) examined the search results independently. Disagreements were reconciled by a third reviewer (M. de Bruin) and through consensus.

\section{Data extraction}

Two coders (A.L. Dima and O. Cunillera) extracted information on: study characteristics (objectives, methodology, country, language, setting, sample size, age, sex, asthma severity and type of inhaled medication studied); adherence behaviours and determinants (definition, measurement and psychometrics); and statistical data (type of analysis and results reported). The data extraction procedure was piloted on articles not included in the review. Each coder extracted data from $50 \%$ of the papers. The accuracy of the recorded information was verified by the other coder, and disagreements were discussed and reconciled.

\section{Quality rating}

Two coders (A.L. Dima and G. Hernandez) rated methodological quality based on six criteria adapted from the STROBE (Strengthening the Reporting of Observational Studies in Epidemiology) guidelines, which are considered key requirements for observational studies [25, 26]. Scoring was performed on a four-level response format, from no information reported to adequate reporting of appropriately used methodology (Supplementary material 2). The studies were judged on clarity of methods and pertinence in six domains: 1) selection of participants (e.g. sampling strategy, eligibility criteria and methods for assessing eligibility); 2) definition of variables (i.e. outcomes, determinants and confounders); 3) description of data sources and measurement procedures for all variables; 4) addressing potential sources of bias (e.g. medical surveillance, recall, or response bias); 5) sample size justification (e.g. power analysis, multiple comparisons correction); and 6) data analysis (e.g. data preparation, controlling for confounding and data collection, and sensitivity analyses). Disagreements were discussed and reconciled.

\section{Data analysis}

The data on study characteristics and adherence measurement were summarised descriptively. The results on the relationships between adherence determinants and behaviours were grouped separately for reliever (e.g. short-acting $\beta_{2}$-agonists $(\mathrm{SABA})$ ) and controller (e.g. ICS) medication as they relate to different recommendations (daily versus as needed use). Controller adherence was examined separately for the three 
stages of adherence [27]: 1) starting treatment (initiation); 2) accuracy of medication use (implementation); and 3) continuing treatment (persistence). Determinants were classified using the five dimensions of the World Health Organization (WHO) taxonomy [26, 27]: 1) social and economic factors, 2) healthcare team and system-related factors, 3) condition-related factors, 4) therapy-related factors, and 5) patient-related factors; each with additional sub-dimensions. We summarised results regarding the statistical significance and direction of relationships for all studies. Adjusted results obtained by multivariate analyses were prioritised over unadjusted when available.

Metric properties of the six study quality items were investigated. Reliability was assessed by estimating inter-rate agreement with weighted kappa, considered appropriate for ordinal scores [28], and interpreted based on established thresholds for poor, fair, moderate, good and excellent agreement $(0.20,0.40,0.60$ and 0.80) [29]. A Mokken scaling and correlational analyses were performed on consensus scores to evaluate structural validity and examine the relationships between criteria. Total quality scores were computed adding scores on the criteria with adequate metric properties; studies were classified as higher versus lower quality via median split. Statistical analyses were performed with SPSS version 21 (IBM Corp., Armonk, NY, USA) and the R-project (www.R-project.org) mokken package [30, 31].

\section{Results}

\section{Study selection}

The database search identified 2878 unique articles (fig. 1). The two coders agreed on the selection of 213 articles as potentially relevant (Cohen's $\kappa=0.60$ ). The third coder reviewed 235 disagreements and selected 86 additional articles. Thus, 299 articles were reviewed to confirm they fulfilled all inclusion criteria. 213 articles were excluded based on title and abstract, and a further 35 articles were excluded after full manuscript examination. Finally, 51 studies were included in the review. The reasons for exclusion are presented in figure 1.

\section{Study characteristics}

Characteristics of studies are showed in table 1. Most studies were conducted in European countries $(n=22)$ or the USA $(n=19)$. Settings of studies were diverse, and included: primary and secondary care; pharmacies; general population; and various prescription and insurance claims databases. 11 studies used existing databases, while 40 studies collected data directly from patients. 32 studies focused solely on adults (aged >18 years), while 19 studies included adults and children. Sample sizes ranged from 34 to 292738 participants (median (interquartile range) 204 (906)). Most studies included more females than males. Asthma severity was reported in 16 studies and ranged from mild to severe asthma.

\section{Identification}

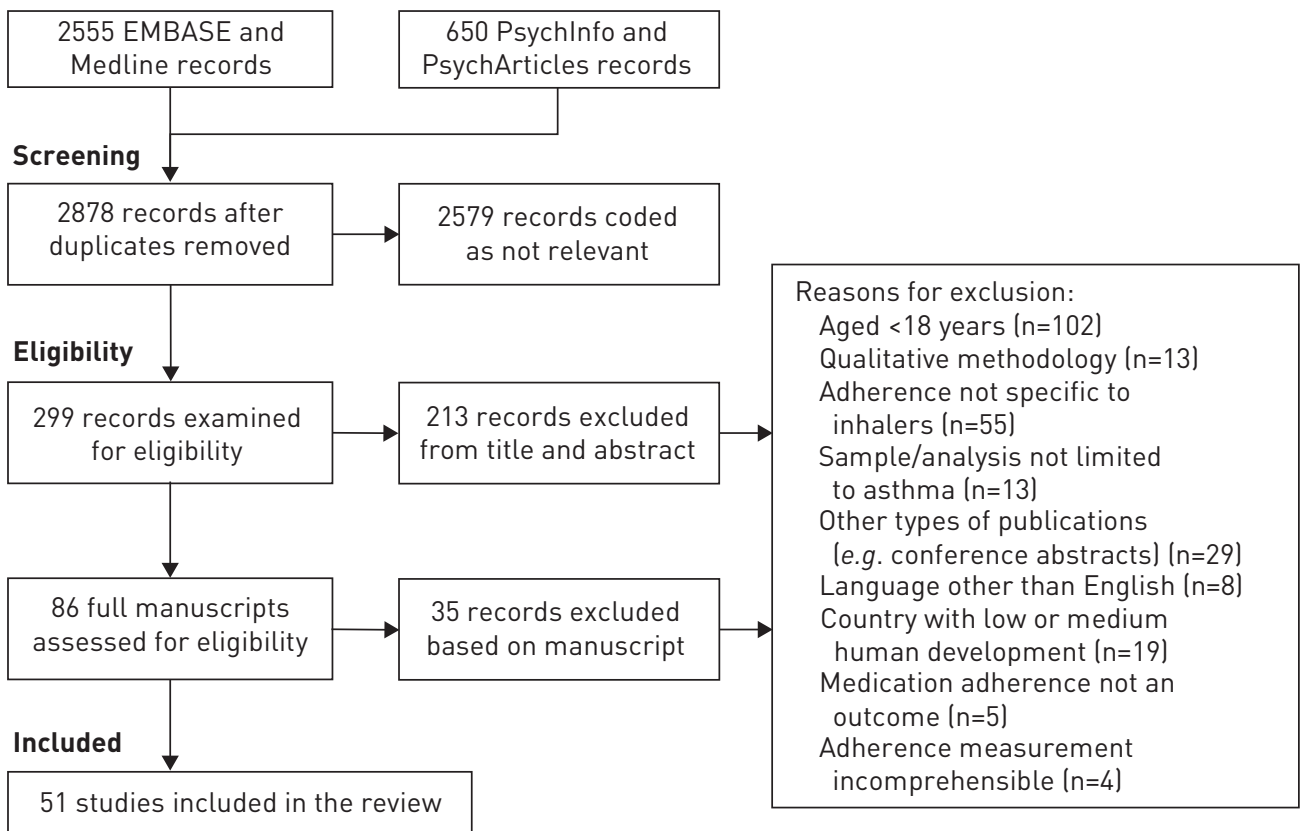

FIGURE 1 Flow diagram of article selection process. 
TABLE 1 Study characteristics of empirical studies on inhaled medication adherence in adults with asthma

\begin{tabular}{|c|c|c|c|c|c|c|c|c|c|c|}
\hline First author [ref.] & Country & Year & Objectives & Study design & Data sources & $\begin{array}{l}\text { Sample } \\
\text { size } n\end{array}$ & Age years & Females & $\begin{array}{l}\text { Asthma } \\
\text { severity: } \\
\text { FEV } 1 \%\end{array}$ & $\begin{array}{l}\text { Inhaled } \\
\text { medication }\end{array}$ \\
\hline Tettersell [32] & UK & 1993 & $\begin{array}{l}\text { Relationship between } \\
\text { knowledge and treatment } \\
\text { adherence }\end{array}$ & $\begin{array}{l}\text { Cross-sectional } \\
\text { (ASD) }\end{array}$ & Primary care & 100 & $50.1 \pm 20.6$ & $9 \%$ & $\begin{array}{l}\text { Moderate } \\
\text { to severe }\end{array}$ & NR \\
\hline BosLey [33] & UK & 1995 & $\begin{array}{c}\text { Psychological factors related } \\
\text { to asthma self-reported care } \\
\text { and compliance }\end{array}$ & Prospective (DPA) & $\begin{array}{l}\text { Primary care and } \\
\text { outpatient clinic }\end{array}$ & 72 & $45 \pm 15$ & $n=62$ & NR & $\begin{array}{l}\text { ICS+LABA, } \\
\text { ICS/LABA }\end{array}$ \\
\hline APTER [34] & USA & 1998 & $\begin{array}{l}\text { Patient characteristics } \\
\text { related to adherence to } \\
\text { twice daily ICS treatment }\end{array}$ & $\begin{array}{l}\text { Prospective (DPA } \\
\text { and ASD) }\end{array}$ & Outpatient clinics & 50 & $46 \pm 14$ & $n=37(74 \%)$ & $75 \pm 21$ & ICS \\
\hline BenNett [35] & UK & 1998 & $\begin{array}{l}\text { Associations between } \\
\text { protection motivation theory } \\
\text { factors (health threat, } \\
\text { outcome, self-reported } \\
\text { efficacy) and adherence to } \\
\text { preventive ICS use }\end{array}$ & $\begin{array}{l}\text { Cross-sectional } \\
\text { (ASD) }\end{array}$ & Primary care & 71 & $47 \pm 19.25$ & $n=40$ & NR & ICS \\
\hline Chambers [36] & USA & 1999 & $\begin{array}{l}\text { Factors associated with } \\
\text { regular ICS use }\end{array}$ & $\begin{array}{l}\text { Cross-sectional } \\
\text { (ASD) }\end{array}$ & Primary care & 394 & Median: 36 & $75 \%$ & NR & ICS \\
\hline Schmaling [37] & USA & 2000 & $\begin{array}{l}\text { Development of measures to } \\
\text { assess psychological factors } \\
\text { important to adherence with } \\
\text { medication regimens }\end{array}$ & $\begin{array}{l}\text { Cross-sectional } \\
\text { (ASD) }\end{array}$ & $\begin{array}{l}\text { Private asthma } \\
\text { clinic and } \\
\text { hospital }\end{array}$ & 53 & $36.1 \pm 9.6$ & $62.3 \%$ & NR & $\begin{array}{l}\text { ICS, LABA, } \\
\text { SABA }\end{array}$ \\
\hline HoRne [38] & UK & 2002 & $\begin{array}{l}\text { Relationship between } \\
\text { reported adherence to } \\
\text { preventer medication and } \\
\text { perceptions and asthma } \\
\text { medication }\end{array}$ & $\begin{array}{l}\text { Cross-sectional } \\
\text { (ASD) }\end{array}$ & Primary care & 100 & $49.3 \pm 18.1$ & $61 \%$ & NR & NR \\
\hline VAN SchaYck [39] & Netherlands & 2002 & $\begin{array}{l}\text { Influence of inhalation } \\
\text { device, patients' inhaler } \\
\text { perceptions, daily frequency, } \\
\text { and duration of treatment on } \\
\text { medication compliance }\end{array}$ & $\begin{array}{l}\text { Prospective (DPA } \\
\text { and ASD) }\end{array}$ & Primary care & 34 & $37 \pm 13$ & $n=19$ & NR & $\begin{array}{l}\text { LABA or } \\
\text { SABA }\end{array}$ \\
\hline APter [40] & USA & 2003 & $\begin{array}{l}\text { Barriers to adherence as } \\
\text { explanations of racial-ethnic } \\
\text { differences in adherence }\end{array}$ & $\begin{array}{l}\text { Prospective (DPA } \\
\text { and ASD) }\end{array}$ & $\begin{array}{l}\text { Primary and } \\
\text { secondary care }\end{array}$ & 85 & $47 \pm 15$ & $\mathrm{n}=61(72 \%)$ & $65 \pm 19$ & ICS \\
\hline JESSOP [41] & UK & 2003 & $\begin{array}{l}\text { Relationship between } \\
\text { cognitive and emotional } \\
\text { representations of asthma } \\
\text { and adherence to inhaled } \\
\text { preventative asthma } \\
\text { medication }\end{array}$ & $\begin{array}{l}\text { Cross-sectional } \\
\text { (ASD) }\end{array}$ & Primary care & 330 & $57.2 \pm 17.9$ & $\begin{array}{c}n=204 \\
(61.8 \%)\end{array}$ & NR & NR \\
\hline LABRECQUE [42] & Canada & 2003 & $\begin{array}{c}\text { Assess compliance to } \\
\text { asthma guidelines and } \\
\text { influence of age on SABA } \\
\text { utilisation }\end{array}$ & Retrospective (ASD) & $\begin{array}{l}\text { Health insurance } \\
\text { database claims }\end{array}$ & 987 & $\begin{array}{l}\text { Range: } \\
5-45\end{array}$ & NR & $\begin{array}{l}\text { Severe } \\
\text { asthma } \\
\text { excluded }\end{array}$ & $\begin{array}{l}\text { SABA (with } \\
\text { or without } \\
\text { ICS) }\end{array}$ \\
\hline
\end{tabular}


TABLE 1 Continued

\begin{tabular}{|c|c|c|c|c|c|c|c|c|c|c|}
\hline First author [ref.] & Country & Year & Objectives & Study design & Data sources & $\begin{array}{l}\text { Sample } \\
\text { size } n\end{array}$ & Age years & Females & $\begin{array}{l}\text { Asthma } \\
\text { severity: } \\
\text { FEV } 1 \%\end{array}$ & $\begin{array}{l}\text { Inhaled } \\
\text { medication }\end{array}$ \\
\hline Nishiyama [43] & UK & 2003 & $\begin{array}{l}\text { Determine if the Jones } \\
\text { Morbidity Index can be used } \\
\text { in community pharmacy to } \\
\text { identify those who have poor } \\
\text { control }\end{array}$ & $\begin{array}{c}\text { Cross-sectional } \\
\text { (ASD) }\end{array}$ & $\begin{array}{l}\text { Pharmacy } \\
\text { database }\end{array}$ & 306 & $38.5 \pm 20.6$ & $54.5 \%$ & NR & $\begin{array}{l}\text { ICS and } \\
\text { SABA }\end{array}$ \\
\hline BALKRISHNAN [44] & USA & 2005 & $\begin{array}{l}\text { Asthma-related healthcare } \\
\text { costs, medication } \\
\text { adherence, ICS and newly } \\
\text { started on MON versus SAL }\end{array}$ & Retrospective (DPA) & $\begin{array}{l}\text { Health insurance } \\
\text { database claims }\end{array}$ & 198 & $\begin{array}{c}22 \pm 19.5 \\
\text { MON } \\
24 \pm 18.2 \mathrm{SAL}\end{array}$ & $\begin{array}{c}52.5 \% \\
\text { MON } \\
59.8 \% \text { SAL }\end{array}$ & NR & $\begin{array}{l}\text { ICS+LABA } \\
\text { versus ICS } \\
\quad+\text { MON }\end{array}$ \\
\hline LACASSE [45] & Canada & 2005 & $\begin{array}{l}\text { Describe patterns of } \\
\text { compliance and identify } \\
\text { factors determining the } \\
\text { compliance to ICS in adults }\end{array}$ & $\begin{array}{l}\text { Prospective (DPA } \\
\text { and ASD) }\end{array}$ & NR & 124 & $47 \pm 15$ & $n=73$ & $\begin{array}{l}\text { Mild- } \\
\text { moderate }\end{array}$ & ICS \\
\hline Stempel [46] & USA & 2005 & $\begin{array}{l}\text { Patient adherence with } \\
\text { several medication } \\
\text { regimens: FP/SAL, FP+SAL, } \\
\text { FP+MON, FP, MON }\end{array}$ & Retrospective (ASD) & $\begin{array}{l}\text { Health insurance } \\
\text { database claims }\end{array}$ & 3503 & $38.7 \pm 17$ & $64.5 \%$ & NR & $\begin{array}{l}\text { ICS, LABA, } \\
\text { MON }\end{array}$ \\
\hline BENDER [47] & USA & 2006 & $\begin{array}{l}\text { Factors related to refill } \\
\text { adherence to FP/SAL }\end{array}$ & Retrospective (ASD) & $\begin{array}{l}\text { Pharmacy } \\
\text { database }\end{array}$ & 5504 & $54 \pm 22$ & $60.2 \%$ & NR & ICS/LABA \\
\hline Chatkin [48] & Brazil & 2006 & $\begin{array}{l}\text { Rate of compliance with } \\
\text { preventive treatment for } \\
\text { moderate and severe } \\
\text { persistent asthma }\end{array}$ & Prospective (DPA) & Primary care & 131 & $44.4 \pm 16.6$ & $71 \%$ & $\begin{array}{c}\text { Severe } \\
\text { persistent }\end{array}$ & ICS/LABA \\
\hline Hasegawa [49] & Japan & 2006 & $\begin{array}{l}\text { Comparison between } \\
\text { compliance to FP diskus } \\
\text { versus FP diskhaler }\end{array}$ & Retrospective (ASD) & $\begin{array}{l}\text { Pharmacy } \\
\text { database }\end{array}$ & 337 & $\begin{array}{l}54.2 \pm 16.8 \mathrm{FP} \\
\text { diskhaler } \\
57.7 \pm 18.2 \\
\text { FP diskus }\end{array}$ & $\begin{array}{l}\text { 56.3\% FP } \\
\text { diskhaler } \\
57 \% \text { FP } \\
\text { diskus }\end{array}$ & NR & ICS \\
\hline Marceau [50] & Canada & 2006 & $\begin{array}{l}\text { Compare persistence, } \\
\text { adherence and effectiveness } \\
\text { between patients with } \\
\text { asthma starting combination } \\
\text { or concurrent therapies (ICS } \\
\text { and LABA) }\end{array}$ & $\begin{array}{l}\text { Prospective (DPA } \\
\text { and ASD) }\end{array}$ & $\begin{array}{l}\text { Health insurance } \\
\text { database claims }\end{array}$ & 5118 & $32.6 \pm 8.2$ & $63.3 \%$ & NR & $\begin{array}{l}\text { ICS/LABA } \\
\text { versus ICS } \\
+ \text { LABA }\end{array}$ \\
\hline Онм [51] & USA & 2006 & $\begin{array}{l}\text { Explore asthma symptom } \\
\text { perception and its } \\
\text { relationship with adherence } \\
\text { to asthma treatment }\end{array}$ & $\begin{array}{c}\text { Cross-sectional } \\
\text { (ASD) }\end{array}$ & $\begin{array}{l}\text { Asthma/allergy } \\
\text { clinics }\end{array}$ & 120 & $44.8 \pm 9.27$ & $78 \%$ & $\begin{array}{l}\text { Mild to } \\
\text { severe }\end{array}$ & ICS \\
\hline Tavasol [52] & Ireland & 2006 & $\begin{array}{l}\text { Factors related to patients' } \\
\text { compliance with prescribed } \\
\text { metered dose inhaler drugs }\end{array}$ & $\begin{array}{l}\text { Cross-sectional } \\
\text { (ASD) }\end{array}$ & $\begin{array}{l}\text { Outpatient } \\
\text { department }\end{array}$ & 160 & $47.67 \pm 12.78$ & $\begin{array}{c}n=105 \\
(65.6 \%)\end{array}$ & NR & $\begin{array}{l}\text { ICS, LABA, } \\
\text { SABA }\end{array}$ \\
\hline ULRIK [53] & Denmark & 2006 & $\begin{array}{l}\text { Patient-related aspects of } \\
\text { adherence among adult } \\
\text { asthmatics }\end{array}$ & $\begin{array}{c}\text { Cross-sectional } \\
\text { (ASD) }\end{array}$ & $\begin{array}{l}\text { Community } \\
\text { (web-based panel } \\
\text { for market } \\
\text { research) }\end{array}$ & 509 & Range: 18-45 & $\begin{array}{l}n=317 \\
(62 \%)\end{array}$ & $\begin{array}{c}\text { Mild: } 77 \% \\
\text { Moderate: } \\
12 \% \\
\text { Severe: } \\
11 \%\end{array}$ & $\begin{array}{l}\text { ICS, ICS } \\
+ \text { LABA }\end{array}$ \\
\hline
\end{tabular}




\begin{tabular}{|c|c|c|c|c|c|c|c|c|c|c|}
\hline First author [ref.] & Country & Year & Objectives & Study design & Data sources & $\begin{array}{l}\text { Sample } \\
\text { size } n\end{array}$ & Age years & Females & $\begin{array}{l}\text { Asthma } \\
\text { severity: } \\
\text { FEV } 1_{1} \%\end{array}$ & $\begin{array}{l}\text { Inhaled } \\
\text { medication }\end{array}$ \\
\hline WiLLiams [54] & USA & 2007 & $\begin{array}{l}\text { Factors associated with ICS } \\
\text { adherence among patients } \\
\text { with asthma, and among } \\
\text { African-American and white } \\
\text { patients separately }\end{array}$ & Retrospective (ASD) & $\begin{array}{l}\text { Health } \\
\text { maintenance } \\
\text { organisation }\end{array}$ & 176 & $40.8 \pm 7.7$ & $\begin{array}{l}n=115 \\
(68.1 \%)\end{array}$ & NR & ICS \\
\hline WiLliams [55] & USA & 2007 & $\begin{array}{l}\text { Estimate rates of primary } \\
\text { nonadherence and explore } \\
\text { associated factors }\end{array}$ & Retrospective (ASD) & $\begin{array}{l}\text { Health } \\
\text { maintenance } \\
\text { organisation }\end{array}$ & 1064 & $31.9 \pm 16.5$ & $59.8 \%$ & NR & ICS \\
\hline BREeKVeLdt-Postma [56] & Netherlands & 2008 & $\begin{array}{l}\text { Determinants of persistence } \\
\text { with ICS }\end{array}$ & Prospective (DPA) & $\begin{array}{l}\text { Pharmacy } \\
\text { database }\end{array}$ & 5563 & Range: 0-34 & $\begin{array}{l}51.5- \\
57.2 \%\end{array}$ & NR & $\begin{array}{l}\text { ICS, ICS } \\
+ \text { LABA }\end{array}$ \\
\hline JANSON [57] & USA & 2008 & $\begin{array}{l}\text { Describe asthma medication } \\
\text { adherence, identify } \\
\text { predictors of ICS underuse } \\
\text { and SABA or LABA overuse }\end{array}$ & $\begin{array}{c}\text { Cross-sectional (DPA } \\
\text { and ASD) }\end{array}$ & $\begin{array}{l}\text { Primary and } \\
\text { secondary care } \\
\text { (random-digit } \\
\text { dialling) }\end{array}$ & 158 & $\begin{array}{c}\text { 48.7 } \pm 7.4 \text { ICS } \\
\text { adherent, } \\
46.7 \pm 8.5 \text { ICS } \\
\text { non-adherent, } \\
46.5 \pm 8.8 \text { SABA } \\
\text { adherent, } \\
46.2 \pm 7.3 \text { SABA } \\
\text { over use }\end{array}$ & $68 \%$ & NR & $\begin{array}{l}\text { ICS and } \\
\text { SABA or } \\
\text { LABA }\end{array}$ \\
\hline Martínez-Moragón [58] & Spain & 2008 & $\begin{array}{l}\text { Relationship between failure } \\
\text { to perceive dyspnoea } \\
\text { associated with bronchial } \\
\text { obstruction and treatment } \\
\text { nonadherence in asthmatic } \\
\text { patients }\end{array}$ & $\begin{array}{l}\text { Cross-sectional } \\
\text { (ASD) }\end{array}$ & $\begin{array}{l}\text { Outpatient } \\
\text { respiratory clinics }\end{array}$ & 48 & $\begin{array}{l}\text { 45: range } \\
30-60\end{array}$ & $50 \%$ & Moderate & ICS/LABA \\
\hline $\operatorname{McG}_{A N N}[59]$ & USA & 2008 & $\begin{array}{l}\text { Relationship between denial } \\
\text { of illness and compliance } \\
\text { with inhaled controller } \\
\text { asthma medications }\end{array}$ & Prospective (DPA) & $\begin{array}{l}\text { Asthma clinics, } \\
\text { advertisements, } \\
\text { local college }\end{array}$ & 51 & $\begin{array}{c}42 \pm 14.99 ; \text { range: } \\
18-68\end{array}$ & $82.3 \%$ & NR & $\begin{array}{c}\mathrm{NR} \\
\text { (controller) }\end{array}$ \\
\hline MeNCKeBERg [60] & Netherlands & 2008 & $\begin{array}{l}\text { Relationship between beliefs } \\
\text { about ICS (necessity and } \\
\text { concerns) and adherence }\end{array}$ & $\begin{array}{l}\text { Cross-sectional/ } \\
\text { retrospective (ASD) }\end{array}$ & $\begin{array}{l}\text { Pharmacy } \\
\text { database }\end{array}$ & 238 & $36.2 \pm 6.3$ & $67 \%$ & $N R$ & ICS \\
\hline Wells [61] & USA & 2008 & $\begin{array}{l}\text { Factors that contribute to } \\
\text { ICS adherence among } \\
\text { African-American and white } \\
\text { adults with asthma }\end{array}$ & Retrospective (ASD) & $\begin{array}{l}\text { Health } \\
\text { maintenance } \\
\text { organisation }\end{array}$ & 1006 & $43.1 \pm 10.4$ & $\begin{array}{l}\mathrm{n}=716 \\
(71.2 \%)\end{array}$ & NR & ICS \\
\hline AXeLsSON [62] & Sweden & 2009 & $\begin{array}{l}\text { Personality traits related to } \\
\text { asthma control, } \\
\text { health-related quality of life } \\
\text { and adherence to regular } \\
\text { asthma medication }\end{array}$ & $\begin{array}{l}\text { Cross-sectional } \\
\text { (ASD) }\end{array}$ & $\begin{array}{l}\text { Epidemiological } \\
\text { study }\end{array}$ & 109 & Range: $21-23$ & $61.6 \%$ & NR & $\begin{array}{l}\text { ICS/LABA, } \\
\text { ICS, LABA, } \\
\text { SABA }\end{array}$ \\
\hline
\end{tabular}




\begin{tabular}{|c|c|c|c|c|c|c|c|c|c|c|}
\hline First author [ref.] & Country & Year & Objectives & Study design & Data sources & $\begin{array}{l}\text { Sample } \\
\text { size } n\end{array}$ & Age years & Females & $\begin{array}{l}\text { Asthma } \\
\text { severity: } \\
\text { FEV } 1 \%\end{array}$ & $\begin{array}{c}\text { Inhaled } \\
\text { medication }\end{array}$ \\
\hline$B_{A E}[63]$ & South Korea & 2009 & $\begin{array}{l}\text { Baseline information about } \\
\text { ICS adherence in Korea, } \\
\text { factors related to ICS } \\
\text { adherence, clinical } \\
\text { implications of ICS } \\
\text { adherence for asthma } \\
\text { control }\end{array}$ & $\begin{array}{l}\text { Cross-sectional/ } \\
\text { retrospective (ASD) }\end{array}$ & $\begin{array}{l}\text { Clinical centres in } \\
\text { university } \\
\text { hospitals }\end{array}$ & 185 & NR & NR & NR & $\begin{array}{l}\text { ICS or ICS/ } \\
\text { LABA }\end{array}$ \\
\hline LAFOREST [64] & France & 2009 & $\begin{array}{l}\text { Characteristics of patients } \\
\text { with interruptions of ICS, } \\
\text { intentional or accidental }\end{array}$ & $\begin{array}{l}\text { Cross-sectional } \\
\text { (ASD) }\end{array}$ & $\begin{array}{c}\text { Primary care } \\
\text { database }\end{array}$ & 204 & $53.8 \pm 19.6$ & $59.3 \%$ & All ranges & $\begin{array}{l}\text { ICS only } \\
\text { or in } \\
\text { combination }\end{array}$ \\
\hline Ponieman [65] & USA & 2009 & $\begin{array}{l}\text { Impact of potentially } \\
\text { modifiable medication } \\
\text { beliefs on adherence with } \\
\text { ICS therapy across time }\end{array}$ & $\begin{array}{c}\text { Prospective (DPA and } \\
\text { ASD) }\end{array}$ & $\begin{array}{l}\text { General internal } \\
\text { medicine clinics }\end{array}$ & 261 & $\begin{array}{l}48 \pm 13 ; \\
\text { range 20-87 }\end{array}$ & $82 \%$ & $\begin{array}{l}\text { Persistent } \\
\text { asthma }\end{array}$ & ICS \\
\hline FrIEDMAN [66] & USA & 2010 & $\begin{array}{l}\text { Adherence and asthma } \\
\text { control in adolescents and } \\
\text { young adults with mild } \\
\text { asthma who began } \\
\text { treatment with MF or FP }\end{array}$ & Retrospective (ASD) & $\begin{array}{l}\text { Health insurance } \\
\text { claims database }\end{array}$ & 1384 & $\begin{array}{c}\text { Mean: } 16.3 \text { MF; } \\
\text { 16.5 FP; } \\
\text { range: } 12-25\end{array}$ & $\begin{array}{l}51.3 \% \mathrm{MF} \\
55.3 \% \mathrm{FP}\end{array}$ & Mild & ICS \\
\hline TAKEMURA [67] & Japan & 2010 & $\begin{array}{l}\text { Assess factors and } \\
\text { mechanisms that contribute } \\
\text { to and clinical outcomes } \\
\text { relating to adherence }\end{array}$ & $\begin{array}{l}\text { Cross-sectional } \\
\text { (ASD) }\end{array}$ & Respiratory clinic & 176 & $57 \pm 15$ & $n=89$ & NR & $\begin{array}{l}\text { ICS, ICS/ } \\
\text { LABA }\end{array}$ \\
\hline BoLman [68] & Netherlands & 2011 & $\begin{array}{l}\text { Explain ICS adherence by } \\
\text { the attitude, social influence } \\
\text { and self-efficacy model and } \\
\text { habit strength (moderation } \\
\text { and mediation relationships) }\end{array}$ & $\begin{array}{l}\text { Cross-sectional } \\
\text { (ASD) }\end{array}$ & Pharmacy & 139 & $31.5 \pm 5.6$ & $\begin{array}{c}n=98 \\
(70.5 \%)\end{array}$ & NR & ICS \\
\hline EMILSSON [69] & Sweden & 2011 & $\begin{array}{l}\text { Influence of personality } \\
\text { traits and beliefs about } \\
\text { medicines on asthma } \\
\text { medication adherence }\end{array}$ & $\begin{array}{l}\text { Cross-sectional } \\
\text { (ASD) }\end{array}$ & NR & 35 & $52.8 \pm 14.7$ & $n=25$ & NR & $\begin{array}{l}\text { ICS/LABA, } \\
\text { ICS+LABA, } \\
\text { ICS, LABA }\end{array}$ \\
\hline Small [70] & UK & 2011 & $\begin{array}{c}\text { Relationship between } \\
\text { inhaler satisfaction and } \\
\text { patient compliance Influence } \\
\text { on health and } \\
\text { patient-reported outcomes }\end{array}$ & $\begin{array}{l}\text { Cross-sectional } \\
\text { (ASD) }\end{array}$ & $\begin{array}{l}\text { Specialists' and } \\
\text { primary care }\end{array}$ & 2135 & NR in adults & NR & NR & NR \\
\hline Suzukı [71] & Japan & 2011 & $\begin{array}{l}\text { Associations between } \\
\text { several factors of asthma } \\
\text { therapy (patients adherence, } \\
\text { asthma severity) }\end{array}$ & Retrospective (ASD) & $\begin{array}{l}\text { University } \\
\text { hospital }\end{array}$ & 50 & $36.3 \pm 7.9$ & $46 \%$ & NR & ICS \\
\hline
\end{tabular}




\begin{tabular}{|c|c|c|c|c|c|c|c|c|c|c|}
\hline First author [ref.] & Country & Year & Objectives & Study design & Data sources & $\begin{array}{l}\text { Sample } \\
\text { size } n\end{array}$ & Age years & Females & $\begin{array}{l}\text { Asthma } \\
\text { severity: } \\
\text { FEV1 \% }\end{array}$ & $\begin{array}{l}\text { Inhaled } \\
\text { medication }\end{array}$ \\
\hline Foster [72] & Australia & 2012 & $\begin{array}{c}\text { Identify potentially } \\
\text { modifiable beliefs and } \\
\text { behaviours that predict ICS/ } \\
\text { LABA adherence }\end{array}$ & Prospective (ASD) & $\begin{array}{l}\text { Community } \\
\text { pharmacies, } \\
\text { advertising, } \\
\text { primary care, } \\
\text { volunteer } \\
\text { database }\end{array}$ & 99 & $47.6 \pm 15.8$ & $n=57$ & $83 \% \pm 23 \%$ & ICS/LABA \\
\hline Ahmedani [73] & USA & 2013 & $\begin{array}{l}\text { Relationships between locus } \\
\text { of control factors (God, } \\
\text { doctors, other people, } \\
\text { change and internal) and } \\
\text { ICS adherence }\end{array}$ & $\begin{array}{c}\text { Cross-sectional } \\
\text { (ASD) }\end{array}$ & Primary care & 1025 & $37.6 \pm 14.8$ & $\begin{array}{c}n=675 \\
(65.9 \%)\end{array}$ & $N R$ & ICS \\
\hline AxeLsSon [74] & Sweden & 2013 & $\begin{array}{l}\text { To determine the mediating } \\
\text { effects of medication beliefs } \\
\text { between personality traits } \\
\text { and adherence }\end{array}$ & $\begin{array}{l}\text { Cross-sectional } \\
\text { (ASD) }\end{array}$ & Community & 516 & $47.4 \pm 15.6$ & $60 \%$ & NR & $\begin{array}{c}\text { ICS/LABA, } \\
\text { ICS, LABA, } \\
\text { SABA }\end{array}$ \\
\hline Price [75] & UK & 2013 & $\begin{array}{l}\text { Identify characteristics of } \\
\text { patients who prefer } \\
\text { once-daily controller } \\
\text { regimen }\end{array}$ & Retrospective (ASD) & $\begin{array}{c}\text { Primary care } \\
\text { database }\end{array}$ & 3731 & $\begin{array}{c}45.6 \pm 15 \\
\text { range: } 2-94\end{array}$ & $\begin{array}{l}n=2174 \\
(58.3 \%)\end{array}$ & NR & $\begin{array}{l}\text { ICS, ICS } \\
+ \text { LABA }\end{array}$ \\
\hline Price [76] & UK & 2013 & $\begin{array}{l}\text { Compare real life } \\
\text { effectiveness of extra-fine } \\
\text { and larger particle } \\
\text { beclometasone }\end{array}$ & Case-control (DPA) & $\begin{array}{c}\text { Primary care } \\
\text { databases }\end{array}$ & 30354 & Range: $12-80$ & $\begin{array}{c}n=17808 \\
(58.7 \%)\end{array}$ & NR & ICS \\
\hline Schatz [77] & USA & 2013 & $\begin{array}{c}\text { Develop a questionnaire that } \\
\text { reflects nonadherence risk } \\
\text { and identifies adherence } \\
\text { barriers }\end{array}$ & $\begin{array}{c}\text { Prospective (DPA and } \\
\text { ASD) }\end{array}$ & $\begin{array}{c}\text { Health } \\
\text { maintenance } \\
\text { organisation }\end{array}$ & 420 & $41.6 \pm 9.1$ & $\begin{array}{c}n=280 \\
(66.7 \%)\end{array}$ & NR & ICS, SABA \\
\hline WeLls [78] & USA & 2013 & $\begin{array}{l}\text { Determine whether once } \\
\text { daily dosing is associated } \\
\text { with higher ICS adherence } \\
\text { at least twice daily }\end{array}$ & Retrospective (DPA) & $\begin{array}{c}\text { Health } \\
\text { maintenance } \\
\text { organisation }\end{array}$ & 1302 & $\begin{array}{l}28.2 \pm 15.8 \text { once } \\
\text { daily } 31.6 \pm 16.0 \\
\geqslant \text { twice daily }\end{array}$ & $\begin{array}{c}\mathrm{n}=113 \\
(51.1 \%) \\
\text { once daily } \\
\mathrm{n}=656 \\
(60.7 \%) \geqslant \\
\text { twice daily }\end{array}$ & $\begin{array}{l}\text { Low to } \\
\text { severe }\end{array}$ & ICS \\
\hline BADDAR [79] & Oman & 2014 & $\begin{array}{c}\text { Relationships between } \\
\text { patient compliance, inhaler } \\
\text { technique and asthma } \\
\text { control level }\end{array}$ & $\begin{array}{l}\text { Cross-sectional } \\
\text { (ASD) }\end{array}$ & $\begin{array}{l}\text { University } \\
\text { hospital }\end{array}$ & 218 & $\begin{array}{l}\text { Range: } \\
12-72\end{array}$ & $65.1 \%$ & NR & $\begin{array}{l}\text { ICS, ICS/ } \\
\text { LABA, ICS } \\
+ \text { LABA }\end{array}$ \\
\hline FEDerman [80] & USA & 2014 & $\begin{array}{c}\text { Associations of } \\
\text { self-management } \\
\text { behaviours (e.g. medication } \\
\text { adherence and inhaler } \\
\text { technique) with health } \\
\text { literacy }\end{array}$ & Prospective (DPA) & Outpatient clinics & 433 & $\begin{array}{c}\text { Mean: } 67 ; 45 \% \\
\text { aged } 60-64 \\
39 \% \text { aged } 65- \\
74,16 \% \text { aged } \\
\geqslant 75\end{array}$ & $83.8 \%$ & $\begin{array}{l}\text { Moderate } \\
\text { or severe }\end{array}$ & $\begin{array}{l}\text { ICS only or } \\
\text { in } \\
\text { combination }\end{array}$ \\
\hline
\end{tabular}




\begin{tabular}{|c|c|c|c|c|c|c|c|c|c|c|}
\hline First author [ref.] & Country & Year & Objectives & Study design & Data sources & $\begin{array}{l}\text { Sample } \\
\text { size } n\end{array}$ & Age years & Females & $\begin{array}{l}\text { Asthma } \\
\text { severity: } \\
\text { FEV1 \% }\end{array}$ & $\begin{array}{l}\text { Inhaled } \\
\text { medication }\end{array}$ \\
\hline TAYLOR [81] & UK & 2014 & $\begin{array}{l}\text { To develop an annual } \\
\text { measure of ICS adherence } \\
\text { from prescribing data and } \\
\text { statistically model ICS } \\
\text { adherence controlling for } \\
\text { patient factors }\end{array}$ & Retrospective (DPA) & $\begin{array}{l}\text { Primary care } \\
\text { database }\end{array}$ & 292738 & $38.7 \pm 15.4$ & $N R$ & $\begin{array}{l}\text { BTS/SIGN } \\
\text { step 2-5 }\end{array}$ & ICS \\
\hline VAn SteenIs [82] & Netherlands & 2014 & $\begin{array}{l}\text { Relationship between ICS } \\
\text { necessity and concerns, } \\
\text { beliefs and subjectively and } \\
\text { objectively measured } \\
\text { adherence and the } \\
\text { agreement between these } \\
\text { measures }\end{array}$ & $\begin{array}{l}\text { Cross-sectional } \\
\text { (ASD) }\end{array}$ & Pharmacy & 93 & $\begin{array}{c}43.7 \pm 14.5 ; \\
\text { range: } 18-77\end{array}$ & $\begin{array}{c}n=55 \\
(59.1 \%)\end{array}$ & NR & $\begin{array}{l}\text { ICS only or } \\
\text { in } \\
\text { combination }\end{array}$ \\
\hline
\end{tabular}

Data are presented as mean \pm SD, unless otherwise stated. FEV1: forced expiratory volume in $1 \mathrm{~S}$; ASD: adherence simultaneous with determinants measurement; NR: not reported; DPA: determinants preceding adherence measurement; ICS: inhaled corticosteroids; LABA: long-acting $\beta_{2}$-agonists; SABA: short-acting $\beta_{2}$-agonists; MON: montelukast; SAL: salmeterol; FP: fluticasone proprionate; MF: mometasone furoate; BTS: British Thoracic Society; SIGN: Scottish Intercollegiate Guidelines Network. 
20 studies focused on adherence to ICS only, eight assessed adherence to inhaled asthma medication as a generic treatment category and 23 studies focused on various types of medication, including ICS and long-acting $\beta_{2}$-agonists (LABA) or SABA, either in monotherapy or in fixed (ICS/LABA) or free (ICS $+\mathrm{LABA})$ combinations. Two studies analysed repeated measures of adherence in longitudinal cohort designs, prospectively [65] or retrospectively [81]. All other studies collected data cross-sectionally, retrospectively or prospectively $(n=22, n=16$ and $n=12$ studies, respectively) and analysed relationships between determinants and single adherence measures.

There were substantial differences between studies in operationalisation and measurement of both adherence determinants and behaviours (Supplementary material 3). Of the 68 adherence behaviour assessments (several studies used multiple measures) (table 2), 31 relied on patient reports, 24 accessed medical records (prescription and refill data), seven employed electronic monitoring, four used canister weighting, one used dose counters and one requested physician reports. 15 of the patient-reported adherence assessments applied validated questionnaires, such as the Medication Adherence Rating Scale [38] and Revised Asthma Adherence Scale [83], while the remainder used self-constructed nonvalidated questionnaires.

As most results focused on implementation of controller medication, we chose to summarise these both graphically and in the text (figs 2 and 3). The results on controller initiation and persistence and on reliever use were limited and, therefore, are only described textually.

\section{Determinants of controller medication adherence}

Initiation

Determinants of controller initiation were examined in one study that reported a higher probability of non-initiation for younger patients, females, African-American ethnicity (versus white), and with fewer SABA fills in the preceding year [55]. No associations were found with socioeconomic status, comorbidity, costs of treatment and various healthcare utilisation indicators.

\section{Implementation}

We identified 544 results in 47 studies, of which 457 relationships between a determinant and an adherence measure could be assessed in terms of significance and direction of relationship. Figure 2 provides details on the WHO determinant sub-dimensions with at least three results. As different measures of adherence may lead to different associations with determinants, we distinguished between objective measures, medical records and subjective reports with each type of measurement. Results from higher quality studies are presented in figure 3. Determinants with less than three results are only described briefly in the text.

Social and economic factors were investigated in 15 studies. Adherence was related to higher income in three out of eight reported results [34, 40, 53-55, 57-59]; more prescription coverage in one out of four results [34, 40, 45,59]; lower treatment costs in two out of seven results [47, 54, 55, 61, 77]; and lower perceptions of social norms in one out of three results [68, 72, 77]. Several other variables were identified in fewer than three results and were found to be unrelated to adherence: geographical area [47]; urban location [59]; immigration status [52]; crime rate in area of residence [54]; social modelling [68]; and social support [40,68]. Minority status was related to adherence in one result [34], and employment status in one out of two results $[52,59]$.

Eight studies examined healthcare team and system factors, with education provision relating to adherence in three out of four results $[32,45,67]$. Several other variables were examined in fewer than three results: lower adherence was linked to inability to get an appointment when needed in one result [61], to patientprovider communication in one out of two results $[34,40]$, and to the time interval being registered with the same prescriber in one result [81], while receiving a prescription from a specialist versus a generalist was unrelated to adherence [59].

Therapy-related factors were investigated in 18 studies. Adherence was mostly unrelated to the number of drugs in the treatment regimen (three out of four results; $[63,70,78]$ ), the number of daily doses (five out of seven results; [39, 47, 64, 67, 78]), and having reliever inhalers prescribed (four out of five results [34, $47,48,64]$ ). Using dry-powder inhalers (DPIs) versus metered-dose inhalers (MDIs) was linked to adherence in two out of four results $[66,67]$. Some variables examined in a single result were unrelated to adherence: prescribed use of peak flow meter or action plan [45]; treatment duration [67]; using various other drugs [44, 48, 52, 57, 64]; using autohalers versus other MDIs [39]. Other single result variables were related to higher adherence: using diskus DPIs versus diskhaler DPIs [49]; using ultrafine versus large-particle formulation [76]; not using a spacer [52]; and receiving more refills in a prescription [47]. Three studies compared ICS/LABA regimens with different types of alternative regimens and reported 
TABLE 2 Definition and measurement of adherence behaviours in the studies reviewed

\begin{tabular}{|c|c|c|c|}
\hline First author [ref.] & Year & Adherence definition/term & Assessment method \\
\hline Tettersell [32] & 1993 & Taking inhalers as prescribed & $\begin{array}{l}\text { Patient-reported, } \\
\text { single item }\end{array}$ \\
\hline BOSLEY [33] & 1995 & $\begin{array}{c}\text { Noncompliance; taking }<70 \% \\
\text { of prescribed doses or } \\
\text { omitting all doses for } \\
\geqslant 1 \text { week }\end{array}$ & Electronic monitoring \\
\hline
\end{tabular}

APTER [34]

1998 Use of ICS in the last 35 days

Electronic monitoring
Patient-reported, published scale

Patient-reported, single item
As-needed medication use

Daily medication adherence

HoRne [38]

VAN SCHAYcK [39]

Apter [40]

JESSOP [41]

LABRecoue [42]
2002

2003

\section{Medication adherence}

Medication compliance rate

Use of ICS in the last 42 days
2003 Adherence to preventative inhaled medication in the last 3 months
Canister weighting

Canister weighting

Patient-reported, published scale Canister weighting

Electronic monitoring
Patient-reported, published scale (adapted) Medical (refill) records
Conformity of SABA prescription use with accepted good use criteria
Details

Validity/

reliability

NR

Onalers as prescibed? " four response options: "always", "majority of the time l 8 out of 10 doses)", "about half of the time",

"only during or following an attack"

Turbuhaler Inhalation Computer; computed for two 6-week periods as (no. of doses taken)/(no. of doses prescribed) $\times 100$

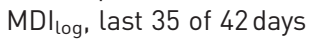
considered, computed for $12-\mathrm{h}$ periods as (recorded - prescribed actuations) $\times 100$; mean truncated adherence computed per subject; dichotomised (<or $>70 \%$ ) RAAS [83]

Item content not specified, four response options: "I use it at least twice a day almost every day", "some days I use it at least twice, but on other days I don't use it at all", I used to use it, but now I don't", "I never used it";

dichotomised into "regular, twice daily" and "less than regular" Total number of medication inhalations for each day in the prescription period

Predicted use (no. days $\times$ no. puff per dayl compared to actual use; computed as percent of prescribed medication used MARS [38]

Medication used as a percentage of medication prescribed $\mathrm{MDI}_{\text {log, }} 42$ days, computed for 12-h periods as (recorded prescribed actuations) $\times 100$; mean truncated adherence computed per subject; divided into four categories $\mid<20 \%, 20-<50 \%$, $50-<75 \%, 75-100 \%)$

RAAS [83] and two extra items on accidental nonadherence

Dichotomous, good use criteria: for SABA with no ICS use, the interval between the targeted SABA prescription and the following refill corresponds to a maximum daily use of two inhalations; for SABA with ICS use, the criterion above, and a daily ICS dose below a fixed threshold 
TABLE 2 Continued

First author [ref.]

Adherence definition/term

Assessment method

Details

Validity/

reliability

\begin{tabular}{|c|c|c|c|c|c|}
\hline \multirow[t]{2}{*}{ Nishiyama [43] } & 2003 & Reliever compliance & $\begin{array}{c}\text { Patient-reported, } \\
\text { interview }\end{array}$ & $\begin{array}{l}\text { Patients were required to state the } \\
\text { drugs and dosage regimens they } \\
\text { used; their reports were compared } \\
\text { with prescription information; } \\
\text { three values were coded: "good"; } \\
\text { "overused"; "underused" (first two } \\
\text { also applied to reliever) }\end{array}$ & NR \\
\hline & & Preventer compliance & $\begin{array}{l}\text { Patient-reported, } \\
\text { interview }\end{array}$ & & NR \\
\hline LACASSE [45] & 2005 & Non-compliance & Electronic monitoring & $\begin{array}{c}\mathrm{MDI}_{\text {log; }} \text { calculated for } 12 \text { weeks } \\
\text { daily as proportion of prescribed } \\
\text { daily dose actually inhaled; } \\
\text { dichotomised as compliant }(>75 \%) \\
\text { or not }\end{array}$ & NR \\
\hline \multirow[t]{3}{*}{ Stempel [46] } & 2005 & Asthma medication refill rate & Medical (refill) records & $\begin{array}{l}\text { Number of } 1 \text {-month supply during } \\
\text { the } 12 \text {-month post-index period }\end{array}$ & NR \\
\hline & & Number of treatment days & Medical (refill) records & $\begin{array}{l}\text { For monotherapy: total days } \\
\text { supplied of medication } \\
\text { For combination: total days } \\
\text { supplied of ICS }\end{array}$ & NR \\
\hline & & SABA refill rates & Medical (refill) records & $\begin{array}{l}\text { Number of } 1 \text {-month supply during } \\
\text { the } 12 \text {-month post-index period }\end{array}$ & NR \\
\hline \multirow[t]{2}{*}{ Bender [47] } & 2006 & Adherence to ICS/LABA & Medical (refill) records & $\begin{array}{l}\text { Total days supplied during } \\
\text { follow-up period }\end{array}$ & NR \\
\hline & & Persistence & Medical (refill) records & $\begin{array}{l}\text { Time to discontinuation computed } \\
\text { as number of days from index date } \\
\text { to date preceding the } \\
\text { pre-specified gap when supply } \\
\text { was exhausted }\end{array}$ & NR \\
\hline Marceau [50] & 2006 & $\begin{array}{l}\text { Persistence versus } \\
\text { discontinuation: having } \\
\text { prescriptions continuously } \\
\text { renewed within the period }\end{array}$ & Medical (refill) records & $\begin{array}{l}\text { Computed as the sum of three } \\
\text { times the duration of the current } \\
\text { prescription (in days) plus all } \\
\text { overlaps accumulated since } \\
\text { therapy start; discontinuation date } \\
\text { was the end date of the last filled } \\
\text { prescription plus all overlaps }\end{array}$ & NR \\
\hline \multirow[t]{2}{*}{ Онм [51] } & 2006 & Use of ICS+LABA & Electronic monitoring & $\begin{array}{l}\text { Advair diskhaler; computed as } \\
\text { (number of counted doses)/ } \\
\text { (number of prescribed doses) } \times 100 \text {; } \\
\text { dichotomised as good adherence } \\
(\geqslant 80 \% \text { ) or not }\end{array}$ & NR \\
\hline & & Medication adherence & $\begin{array}{l}\text { Patient-reported, } \\
\text { published scale }\end{array}$ & MARS [38] & NR \\
\hline
\end{tabular}


TABLE 2 Continued

First author [ref.]

Year Adherence definition/term

Assessment method

Details

Validity/

reliability

\begin{tabular}{ccc}
\hline TAVASOLI [52] & $\begin{array}{c}\text { Compliance to prescribed } \\
\text { MDI drugs }\end{array}$ & $\begin{array}{c}\text { Patient-reported, } \\
\text { interview }\end{array}$
\end{tabular}

ULRIK [53]

2006

Intentional nonadherence

Patient-reported, single item

Adherence

WiLLIAMS [54]

2007

ICS adherence

Williams [55]

2007

Primary non-adherence

ICS adherence

Breekveldt-Postma [56]

2008

Persistence during the first year

JANSON [57]

2008 ICS nonadherence during the last 14 days

SABA or LABA overuse

Four items: "do you use your prescribed spray (MDI drug) regularly?", "have you ever had any history of not using your spray?", "do you still use your last prescribed spray?", "how do you use your spray? Show me"; response scales from 0 to 4 One item: "how often do you decide not to take your controller medication?"; five response options: "almost every day",

"a couple of times every week",

"a couple of times every month",

"a couple of times every year", "hardly ever"

Medical (refill) records

Medical (refill) records

Medical (refill) records

Patient-report, interview

Patient-reported, interview
Patient-reported single item

Item not specified; responses

reported on a three-level scale: taking controller therapy as prescribed, less, or more than prescribed

[Cumulative days supplied]/ (total number of days between refills for 1-year study period); analyses performed also with adherence stratified $(0 \%, 0 \%-80 \%, \geqslant 80 \%)$

No prescription fill information recorded for 3 months after index prescription

Computed as (total days supplied)/ (number of days of observation) $\times 100$; adherence stratified $(0 \%, 0-80 \%, \geqslant 80 \%)$

Computed as number of days from start to time of first failure to continue renewal of initial prescription, based on Inumber of units dispensed)/(number of units to be used per day as defined in pharmacyl

Nursing home assessment of ICS prescription and use, based on inspection of current asthma medication and two questions:

"How many puffs and how many times per day did your doctor tell you to use this?", "During the past 14 days, how many puffs and how many times per day have you used this?"; dichotomised as adherent $1 \geqslant 7$ days of use in previous 14 days) or not

Nursing home assessment on SABA and LABA prescription and use, dichotomised as overuse laverage $>8$ puffs of SABA or $>2$ puffs of LABA -single or combination- per dayl or adherent

NR 
TABLE 2 Continued

\begin{tabular}{llll} 
First author [ref.] & Year & Adherence definition/term & Assessment method \\
\hline MARTínEZ-MoRAGón [58] & 2008 & Frequency of ICS use & $\begin{array}{c}\text { Patient-reported, } \\
\text { single item }\end{array}$
\end{tabular}

McGANn [59]

MencKeberg [60]

2008

2008 "How closely an individual's medication taking behaviours, as measured by the DOSER, approximated prescribed use instructions provided by the healthcare provider" Medication acquisition

Medication adherence

WeLLs [61]

AxeLsSon [62]

BAE [63]

ICS adherence; the proportion of time that the patient had medication available during last 6 months

Medication adherence

Prescription refill adherence

Subjective self-reported adherence

Intentional interruption

Accidental interruption

Ponieman [65]

Friedman [66]

2010
Electronic monitoring

Medical (refill) records published scale Medical (refill) records

Patient-reported, published scale Medical (refill) records

Patient-reported, single item

Patient-reported, single item

Patient-reported, single item

Patient-reported, published scale Medical (refill) records

Medical (refill) records
Patient-reported,

\section{Medication adherence}

Prescription fills

Percentage of days covered
Details

Validity/ reliability

NR adapted after (37); four response options, from "never" to

"at least twice a day almost every day", dichotomised into "almost every day" versus "rarely if ever"

DOSER; ratio of the number of observed correct prescribed use days between day 3 and 14

Agreement with other measures (not specified) $84.32 \%$

(Total days supplied)/(total number of days from first and last refill date) $\times 100$ MARS [38]

$\alpha=0.81$

(Total days supplied)/(number of days of observation) $\times 100$

NR

\section{MARS [38]}

(Number of ICS refills)/12×100; categorised as appropriate use (>80\%), underuse (50-80\%), or extreme underuse $(<50 \%)$

One item: "how often did you take your ICS as prescribed for last 1 year?"; response on a visual analogue scale from $0 \%$ to $100 \%$; categorised as appropriate use $(>80 \%)$, underuse $(50-80 \%)$, and extreme underuse $(<50 \%)$

Six items included: 1) accidental interruption, 2) intentional interruption when feeling better, 3) intentional interruption when feeling worse, 4) reduced use when feeling better, 5) more frequent use of ICS in case of preliminary signs of asthma attack, and 6) intentional changes of doses independently of physician; analyses performed on intentional (when feeling better) and accidental interruption

MARS [38]; dichotomised as good adherence $(\geqslant 4.5)$ or not Total number of prescription refills during the post-index period

(Number of days patients had medication on hand)/(total number of post-index days $=365) \times 100$ 
TABLE 2 Continued

First author [ref.]

Year Adherence definition/term

Assessment method

Details

Validity/

reliability

\begin{tabular}{|c|c|c|}
\hline TAKEMURA [67] & 2010 & $\begin{array}{l}\text { Self-reported adherence to } \\
\text { inhalation regimen }\end{array}$ \\
\hline BoLman [68] & 2011 & Medication adherence \\
\hline EMILSSON [69] & 2011 & Medication adherence \\
\hline SMALL [70] & 2011 & $\begin{array}{l}\text { Physician-perceived } \\
\text { compliance; "the extent to } \\
\text { which the patients are } \\
\text { perceived to follow their } \\
\text { physician's prescribing } \\
\text { instructions and advice" }\end{array}$ \\
\hline Suzukı [71] & 2011 & ICS adherence \\
\hline
\end{tabular}

Patient-reported, published scale (adapted)

FOSTER [72]

2012 Adherence with ICS/LABA

Ahmedani [73]
AxeLsson [74]

2013

ICS adherence

2013

PrICE [75]

Price [76]

2013

ICS adherence

Schatz [77]

2013 Questionnaire low adherence

Percent of days covered

\section{WeLLs [78]}

2013
Medical (prescription

and refill) records

Medical (refill) records

Patient-reported, published scale Patient-reported, published scale

Physician-reported, bespoke scale

Patient-reported, published scale

Patient-report, single item

Medical (prescription and refill) records Patient-reported, published scale Patient-reported, published scale

Medical (prescription) records

Patient-reported, published scale

Medical (prescription and refill) records
Modification of RAAS [83]

NR

concerning the use of inhaled controller medications; mean

adherence score computed; dichotomised as good adherence $(\geqslant 4.0)$ or not MARS [38]

MARS [38]

$\alpha=0.77$

Two items (not specified) on physician-perceived patients' compliance regarding frequency of use and inhaler use; five response options from "not at all compliant" to "fully compliant"

Ratio of doses dispensed in the pharmacy divided by prescribed doses documented in medical charts

Smart inhaler; daily adherence calculated as (no. recorded actuations/no puffs prescribed) $\times 100$, capped at $100 \%$ and averaged for the last 4 weeks of 2 months monitored

Morisky adherence scale [84]

$\alpha=0.92$

Estimation of own inhaler use

(days/week and puffs per day) in the last 4 weeks

(Total days supplied)/(3-month observation period) $\times 100$ MARS [38]

MARS [38], categorised as "low" ("often" or "always" response to any question), "borderline" I'sometimeś responses to $>1$ question), and "good" (any other answer)

(Total days supplied)/(365-day observation period) $\times 100$

Response to "how often are you actually taking your ICS medication now" compared to response to "based on your doctor's most recent instructions, how often were you advised to be taking your ICS medication now" (less frequently)

Days' supply of dispensed canisters over the follow-up at 3 , 6 , and 12 months

Continuous multiple-interval measure of medication availability equals number of days' supply for each fill/total number of days between the present and next fill; averaged for the observation period 
TABLE 2 Continued

\begin{tabular}{|c|c|c|c|c|c|}
\hline First author [ref.] & Year & Adherence definition/term & Assessment method & Details & $\begin{array}{l}\text { Validity/ } \\
\text { reliability }\end{array}$ \\
\hline BADDAR [79] & 2014 & $\begin{array}{c}\text { Compliance with controller } \\
\text { treatment }\end{array}$ & $\begin{array}{l}\text { Interview } \\
\text { cross-checked with } \\
\text { electronic patient } \\
\text { records }\end{array}$ & $\begin{array}{l}\text { Good equals taking } 100 \% \text { of daily } \\
\text { prescribed medication and } \leqslant 2 \\
\text { missed doses/administrations per } \\
\text { week; partial equals taking more } \\
\text { or less than their daily prescribed } \\
\text { medication; poor equals any other } \\
\text { inhaler use patterns }\end{array}$ & NR \\
\hline FEDERMAN [80] & 2014 & ICS adherence & Dose count & $\begin{array}{l}\text { Review of dose counters for all dry } \\
\text { powder inhaler devices during the } \\
\text { first } 3 \text { months and } 30 \text { days after } \\
\text { each new prescription; } \\
\text { dichotomised as }<80 \% \text { and } \geqslant 80 \%\end{array}$ & NR \\
\hline TAYLOR [81] & 2014 & $\begin{array}{c}\text { Adherence to ICS } \\
\text { prescriptions }\end{array}$ & $\begin{array}{l}\text { Medical (prescription) } \\
\text { records }\end{array}$ & $\begin{array}{l}\text { Prescription possession ratio: } \\
\text { (number of days prescribed during } \\
\text { calendar year)/(number of days in } \\
\text { the interval) } \times 100\end{array}$ & NR \\
\hline & & ICS adherence & Medical (refill) records & $\begin{array}{c}\text { Proportion of days covered: } \\
\text { (number of days' supply)/(365 or } \\
\text { truncated if medication gap } \\
\geqslant 182 \text { ) } \times 100 ; \text { dichotomised as }<80 \% \\
\text { and } \geqslant 80 \%\end{array}$ & NR \\
\hline
\end{tabular}

NR: not reported; ICS: inhaled corticosteroids; RAAS: Revised Asthma Adherence Scale; MARS: Medication Adherence Rating Scale; MDI: metered-dose inhaler; SABA: short-acting $\beta_{2}$-agonists; LABA: long-acting $\beta_{2}$-agonists; $\alpha$ : Cronbach's $\alpha$ test.

better adherence to ICS/LABA compared to ICS and/or LABA and/or SABA [62], and compared with ICS in monotherapy or in combination with LABA or montelukast [46], but no differences in intentional or accidental nonadherence between ICS/LABA and ICS+LABA regimens [64].

Condition-related factors were investigated in 26 results, with nonsignificant results regarding asthma duration (nine results [34, 35, 38, 41, 45, 52, 61, 67]), pulmonary function (six out of eight results [34, 40, $45,51,57,58]$ ), and presence of current symptoms (19 out of 22 results $[34,35,41,43,45,48,52,57,58$, $61,62,64,70,79,82])$. Asthma exacerbations showed 13 nonsignificant $[34,40,48,55,57,67,73,81]$, but also five positive $[36,55,73,81]$ and six negative associations $[52,67,70]$ with adherence. Higher health-related quality of life was associated with better adherence in four out of 11 results $[45,57,62,64$, 67, 70], and higher asthma severity was linked to better adherence in five results $[48,68,71,78,81]$, compared to one negative [81] and six nonsignificant results [40, 52, 64, 70, 71].

Patient-related factors were investigated in 40 studies. Patient demographics such as age and sex were included in numerous studies. Older age related to better adherence in 16 out of 28 results [32, 34, 35, 38, 40, $41,45,47,52-55,57,58,61,63,64,67,69,70,72,73,78,81,82]$. Sex showed 24 nonsignificant results [34, $38,40,45,48,52,54,55,57-59,62-64,67,68,70,71,73,79,82]$, with females showing better adherence in three results $[41,47,53]$ and males in another three $[61,72,78]$. Being of white ethnicity was linked with better adherence in five out of 10 results $[40,48,54,55,57,59,61,70,73,78]$, while participants with higher education levels were more adherent in four out of 10 results [34, 38, 40, 45, 48, 52, 53, 57-59].

Few studies found significant roles of variables related to patients' general health status. Smoking status was consistently unrelated to adherence $[40,48,52,57,58,63,64,71]$, as was depression $[40,45,57,58]$. Higher comorbidity was associated with better adherence in two out of eight results $[47,48,54,55,57$, 63], while less healthcare utilisation was linked to better adherence in two out of 11 results [34, 38, 40, 55, $70]$. Asthma knowledge was found to be unrelated to adherence [32, 53], while medication knowledge was reported to be related to adherence in only one out of five results [34, 40, 61, 77]. Asthma beliefs (i.e. perceptions of the asthma impact in terms of severity, consequences, timeline, etc.) showed inconsistent relationships with adherence, with eight positive results $[35,36,41,53,72], 10$ nonsignificant results [35, $38,41,53,57,58]$, and one negative result [38]. 

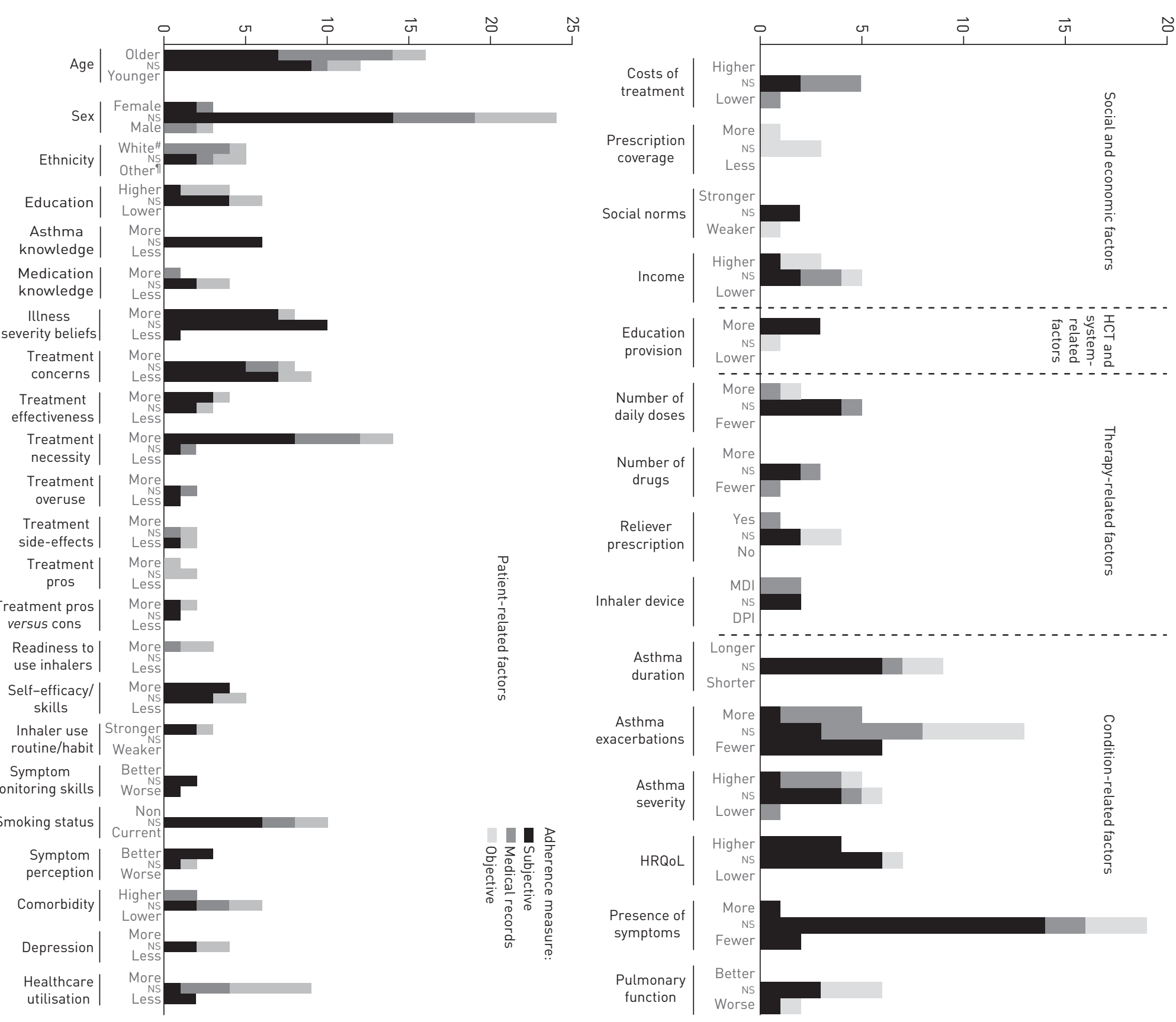

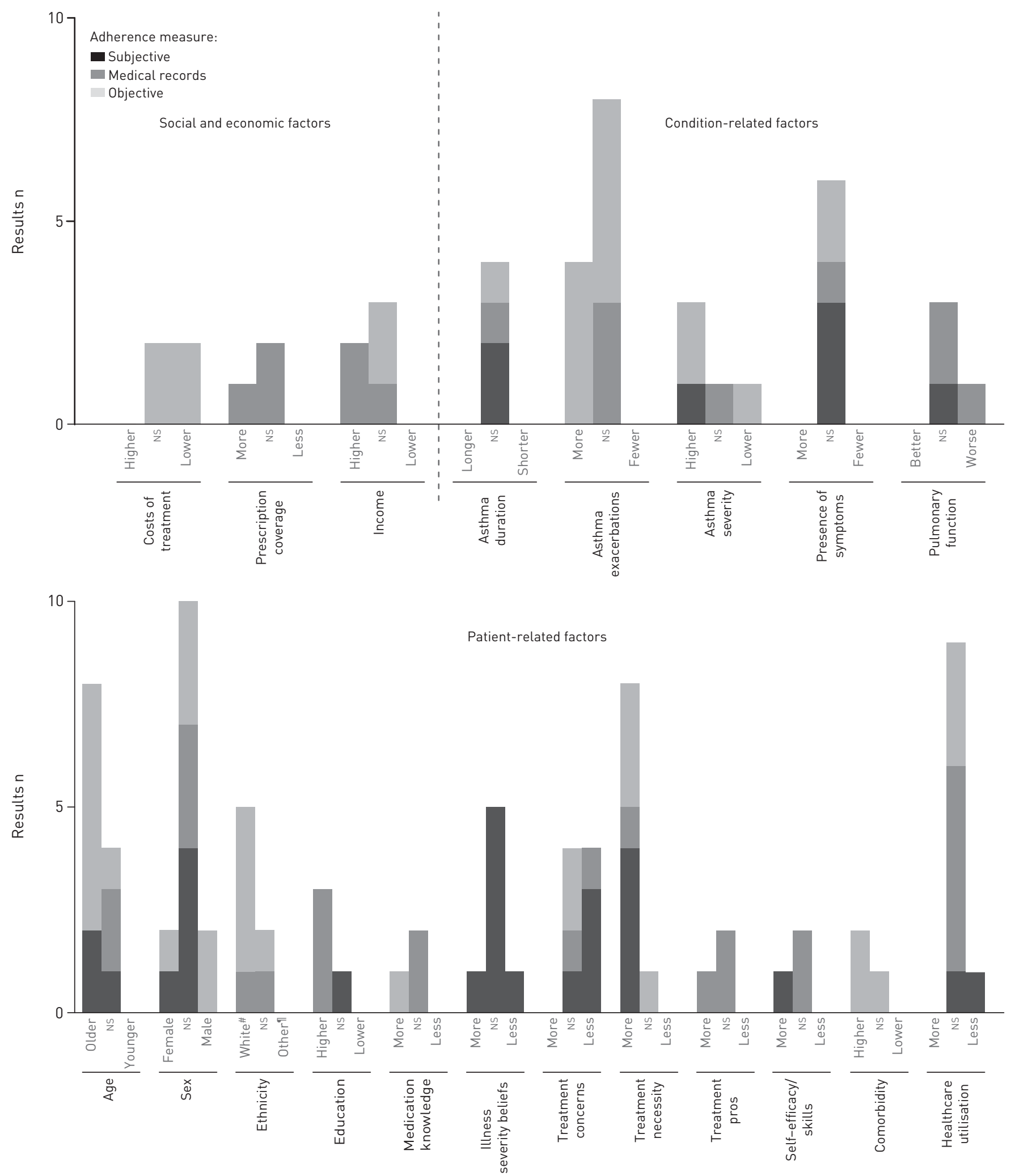

FIGURE 3 Determinants of controller implementation (results from higher quality studies). Number of positive, nonsignificant and negative relationships with adherence indicators for determinants with three or more results identified. Ns: nonsignificant. "*: versus other; ": versus white.

beliefs in inhaler usefulness or benefits in one out of three results [34]. Having fewer concerns about medication was related to better adherence in nine out of 17 results $[38,40,60,64,65,68,72,74]$, lower perceived side-effects in two out of four results $[72,77]$, lower beliefs that medication in general is 
overused in one out of three results $[60,77]$, and stronger beliefs in inhaler necessity relative to concerns in two out of three results $[68,69,72]$. Readiness to use inhalers showed positive associations to adherence in three results $[37,61]$, indicators of self-efficacy in four out of nine results $[32,35,40,57,65,68]$, and stronger adherence routines in three results $[53,68,72]$. A better ability to perceive changes in asthma symptoms was related to adherence in three of five results $[51,58]$, while lower confidence in the ability to monitor symptoms was related to adherence in one of three results $[41,53]$.

Numerous other patient-level variables were examined in fewer than three analyses, most with nonsignificant results: general health status and body mass index [57]; marital status [48]; number of causal attributions for asthma [38]; extent of attributing asthma to internal causes [41]; general health self-efficacy [65]; self-control [45]; and various personality and medical history characteristics [34, 39, 45, $52,58,62,68,69,71,73,74]$. Several exceptions referred to better adherence in people who consider medication as less harmful (two results [60]), display lower neuroticism, higher agreeableness and conscientiousness (one out of two results $[69,74]$ ), and believe more strongly that their asthma can be controlled [38, 41]. Several single results showed better adherence in people with a family history of asthma [71], asthma onset at younger age [58], lower impulsivity [62] and high literacy [80]. Other single findings suggested that more adherent people attribute their asthma more to external factors [41], believe that God is less in control of their health and attribute more control to physicians [73], perceive themselves less vulnerable to side-effects, report higher intention to use inhalers [72], have better inhaler use skills [79], are more satisfied with the device [70], prefer to use inhalers rather than pills [32], have no preferences regarding daily inhaler dosage [75], believe more strongly in participating actively in care [36], and report no symptom improvement due to herbal drugs [52].

\section{Persistence}

Controller-persistence determinants were investigated in three studies, and results are presented below. Patients receiving prescriptions from a specialist, using MDIs, having a lower recommended dose, having once-daily dosing frequency, having used LABAs in the previous year, and having had previous asthma-related hospitalisations were more likely to persist using single ICS treatment during 1 year, while adolescents and patients with more than twice daily dosing frequency were more likely to discontinue [56]. For ICS/LABA therapy, persistence was less likely for adults compared to children, for people with longer therapy duration, higher daily dose, and having used antibiotics in the previous year [56]. Patients using ICS/LABA were more likely to persist with therapy compared to those using ICS+LABA, as were male patients, older patients, those receiving social assistance, those with lower daily dosage, those receiving prescriptions from a specialist, and those using more medications currently and in the previous year [50]. Time to discontinuation of ICS/LABA therapy was longer for male patients, older patients, those paying moderately for treatment, having more refills included in the first prescription, having prescriptions for other conditions, and having had relievers prescribed before the start of the study [47].

\section{Determinants of reliever use}

Reliever use recommendations were examined in three studies. Reliever overuse (as indicator of nonadherence to reliever recommendations) was linked to increased symptoms in two out of three results $[43,57]$, to older age in one out of two results $[42,57]$, and to lower education, higher self-perceived asthma severity and lower general health status in one result [57]. Other factors were unrelated to overuse (e.g. sex, ethnicity, socioeconomic status, smoking status and various health status indicators).

\section{Study quality}

The 51 studies received relatively good quality scores regarding participant selection methods and measurement of variables, with 19 and 14 studies receiving the maximum score, respectively (table 3, Supplementary material 4). Scores were considerably lower on appropriateness of data analysis, measures taken to protect against bias, study size justification and clarity of definitions for the variables included. Common limitations in reporting patient selection were omitting methods of sampling and checking eligibility, and not specifying response rates. The concept definitions often overlapped with the description of measurement methods, or only variable labels were reported. Many studies did not describe measurement methods for all main variables. The majority of studies did not mention any source of bias, and none gave a clear sample size justification or reported optimally on study size decisions. Some studies reported power computations for unspecified analyses, did not correct for multiple comparisons, dichotomised adherence scores without giving a valid rationale, did not control for potential confounders, and offered unclear descriptions of statistical procedures. Inter-rater agreement for the six quality rating criteria (table 3) was poor to moderate, but all discrepancies were resolved through discussion between the two coders. Participant selection methods, measurement of variables, clarity of variable definitions and appropriateness of analyses formed a homogenous scale, with a homogeneity \pm SE of $64 \pm 0.07$. Performance 


\begin{tabular}{|c|c|c|c|c|c|}
\hline Quality criterion & Unknown? & Low $^{+}$ & Medium $^{\S}$ & $\operatorname{High}^{f}$ & Inter-rate agreement weighted $\mathrm{\kappa}$ \\
\hline Participant selection & 0 & 10 & 22 & 19 & 0.41 \\
\hline Definition of variables & 2 & 11 & 35 & 33 & 0.31 \\
\hline Measurement of variables & 0 & 16 & 21 & 14 & 0.38 \\
\hline Addressing sources of bias & 27 & 14 & 8 & 2 & 0.38 \\
\hline Study size & 29 & 19 & 5 & 0 & 0.17 \\
\hline Data analysis & 0 & 24 & 19 & 8 & 0.33 \\
\hline
\end{tabular}

${ }^{\#}:$ n=51. ๆ: no description available; ${ }^{+}$: unclear and/or not appropriate; ${ }^{\S}$ : mostly clear and appropriate, with a few omissions; ${ }^{f}$ : clear and appropriate.

on the two remaining criteria (addressing bias and justifying sample size) was only weakly related to the quality scores on the other four criteria (item properties not shown for brevity).

\section{Discussion}

This systematic review aimed to qualify and synthesise the observational evidence on determinants of inhaled medication adherence in adults with asthma. In the 51 studies included, patient-related factors associated with controller implementation were the most frequently studied, and healthcare team and system factors the least. The more robust evidence linked stronger treatment necessity beliefs to better implementation. The few studies assessing controller initiation and persistence mainly suggest a possible influence of therapy-related factors and patient demographics. Studies on reliever use were scarce, with reliever overuse related to several patient-related factors. This limited evidence offers only provisional guidance for developing inhaler adherence interventions. Furthermore, the findings regarding each adherence determinant and behaviour should be interpreted with caution and within each study context due to the heterogeneity among studies. Our review reveals important knowledge gaps that need to be addressed in the future, and also highlights crucial methodological limitations that can inform researchers regarding concrete steps to take for accumulating sound evidence in future studies.

Regarding the results on determinants of controller use implementation, the substantial focus on patient-related determinants was noted in previous reviews in asthma [19,20] and in other chronic conditions [85-87], and reflects an interest in both identifying at-risk groups and understanding patient perspectives as proximal determinants of patient behaviours. Demographic and clinical characteristics and patients' knowledge of asthma and of medication were generally unrelated to controller use, except a possible higher risk of nonadherence in younger adults. Treatment necessity beliefs were consistently related to better controller implementation but moderate evidence exists on the role of other positive treatment beliefs and concerns. These results confirm a previous review on treatment beliefs [20] and support the relevance of addressing patients' views regarding their condition and treatment in adherence interventions.

Determinant categories not related to patients were studied substantially less and should be prioritised in future research. Condition- and therapy-related factors seemed unrelated to controller implementation behaviours or showed inconsistent results. Among these factors, several medical outcomes, such as asthma exacerbations, severity or symptoms, showed contradictory results, suggesting that their relationships with adherence might vary depending on other parameters, which would need careful examination. Despite the relevance of social and economic factors identified in previous reviews [85-87], only financial information was examined more extensively but showed inconsistent results. Limited data were available on the influence of the social environment in adults with asthma, despite the key role of social factors identified in children's asthma management [19] and in adherence to other long-term treatments for chronic conditions in general $[85,88]$. Healthcare team and system factors were rarely studied, although the improvement of health services for chronic conditions is currently a priority [89] and adherence-enhancing interventions usually include changes in the structure of healthcare delivery [10]. This highlights the need for further research on the structure and content of adherence support in routine clinical care, which can have a major impact on patient behaviours and treatment success rates [90, 91]. Future studies could also benefit from adopting broader theoretical approaches that also explore factors beyond the individual patient level, such as the Precede-Proceed framework, which would facilitate behaviour change intervention design [92].

The barriers to evidence consolidation identified during the present review raise an important question: what methodological standards would future studies apply to obtain quality evidence on determinants of inhaler adherence? Table 4 summarises nine main barriers and several recommendations for improvement, formulated considering the existing methodological advice for observational research [26] and adherence 
TABLE 4 Barriers and recommendations for a solid evidence base on asthma inhaler adherence determinants

Current limitations When conducting a new study

Heterogeneity in variable selection, definition and measurement, study design and statistical analyses

\section{Limited theoretical basis for variable selection and lack of an integrated theoretical approach}

\author{
Lack of robust study designs for causal inferences \\ in most studies
}

\author{
Low or medium quality participant selection \\ in some studies
}

\section{Insufficient description of variable definitions and measurement}

Low quality of measurement

\section{Sources of bias rarely addressed}

Study size rarely addressed

Low or medium quality data analysis procedures in most studies
Consider previous similar studies when selecting determinants and behaviours

Clarify variable definitions in relation to previous studies

Consider using established measures of adherence behaviours and determinants if available

Consider using established study designs and data analysis methods if appropriate

Use existing behavioural theory to select variables

Focus on testing multi-determinant models instead of a few preferred determinants

If testing new models, clarify the choice and relationships with existing theories

Prioritise the use of repeated measure longitudinal designs

Assess adherence determinants prior to behaviours

Choose time lags in which causal influence is likely

Control for other possible causal influences

Use prior literature to decide on clear inclusion criteria that allow comparisons with other studies

Employ systematic procedures for participant selection

Report participant selection procedures clearly and completely

Provide a clear rationale and description for included variables

Provide comprehensive descriptions of measurement tools or methods in the manuscript or supplementary materials

Select or develop psychometrically sound measures

Examine psychometrics as preliminary analyses

Report results of psychometric evaluation

Reflect on possible sources of bias (e.g. response, recall, surveillance bias) and take steps to minimise their effect

Consider the probability of type I and type II errors given the research question, population and resources available

Consult methodological literature relevant for the intended analyses Perform and report on preparatory analyses (e.g. missing data)

Do not group continuous data unless solid justification exists and analyses are performed with both continuous and grouped data

Control for possible confounders and justify their selection

Adjust for sampling strategy and hierarchical data structures research [93] in order to invite further dialogue on this topic. The first barrier identified was the substantial study heterogeneity, not only in sample characteristics but also in variable selection, definition, measurement, study design and statistical analyses. Secondly, the studies lacked a unifying theoretical approach which led to differences in variable selection and, thus, to many determinants being examined only in single studies, often without a theoretical justification. Finally, the results gave limited insight regarding causal influences, as only two studies involved repeated measures of adherence $[65,81]$ and only 17 studies measured determinants before adherence. Moreover, many studies showed limitations in the six quality criteria assessed, although several studies performed well (Supplementary material 4). To address these barriers, we endorse the practical recommendations provided in STROBE [26] and provide brief advice based on STROBE and our experience in this review. Theoretical frameworks and taxonomies of adherence behaviours and determinants are available [27, 94, 95] and should be used more extensively. Conducting research on common theoretical and measurement foundations would allow the field to progress from identifying bivariate or multivariate associations in heterogeneous prediction models towards testing more homogeneous and comprehensive causal models.

Beyond the practical recommendations for future inhaler adherence studies, our review also highlighted the need to develop consensus on several methodological aspects. The fact that few studies reported on variable 
definitions, sources of bias and study size suggests that many researchers might not be aware of their importance for observational studies. The latter two aspects were unrelated to the overall study quality, suggesting that even in higher quality studies, bias and sample size are not systematically considered. More discussion is needed among methodologists and researchers to establish their relevance and specify concrete steps to implement them. These results add to previously expressed concerns regarding the lack of validated tools to evaluate quality in observational studies [23], and highlight a general need for further detailing and clarifying methodological guidelines in this area. Our experience with coding quality exposed the difficulties of assessing these broad criteria given the diversity of designs and brief descriptions permitted by space constraints. We would, therefore, encourage adherence-specific methodological guidelines that can be reported in a standard format as supplementary material in published studies.

Our review has several limitations. First, interpreting the summary based on both adjusted and unadjusted results requires caution, as multivariate analyses control for different sets of confounders, while bivariate analyses ignore any additional influences and may reflect biased relationships. We chose to prioritise adjusted over unadjusted data to avoid this, but we acknowledge that the findings may be biased and we recommend the use of theory-based models to provide more valid and replicable results. Secondly, inter-rate reliability for quality scores was low, which may reflect suboptimal study reporting, difficulty of applying the criteria based on the given definitions, or insufficient training of coders. Although the coders were able to reach consensus, these difficulties illustrate the need for more concrete definitions applicable across studies by coders with diverse research backgrounds. Thirdly, we focused our review on developed nations, as the contribution of determinant dimensions on adherence may be different in developing nations, particularly regarding access to care [86], but only 19 studies were excluded based on this criterion. Finally, meta-analyses were not possible due to the substantial heterogeneity; therefore, we opted for a qualitative summary and for identifying methodological improvements that would make future studies more amenable to meta-analytic approaches.

Our findings suggest that adults with asthma implement controller use recommendations better if they believe more strongly in the necessity of using inhalers, and possibly if they hold other positive beliefs and less concerns about using inhalers. Younger adult patients may be more at risk of nonadherence. Other patient-, condition- and therapy-related factors are either mostly unrelated to adherence or partly studied, and little is known about the role of social, economic and healthcare factors. Initiation and discontinuation of controller use and reliever use behaviours were scarcely explored. Moreover, the methodological limitations identified diminish the strength of current evidence. Our key recommendations for further research are to improve methodology and use established theoretical frameworks, which should enable the development of a cumulative evidence base of causes of nonadherence to asthma inhalers among adults.

\section{Acknowledgements}

We would like to thank Eric van Ganse (Claude Bernard University Lyon 1, Lyon, France) and Marcel Bouvy (Utrecht University, Utrecht, the Netherlands) for valuable discussions regarding the systematic review process, and Dan Dediu (Max Plank Institute, Nijmegen, the Netherlands) for support with conducting the review and summarising results visually.

\section{References}

1 British Thoracic Society, Scottish Intercollegiate Guidelines Network. British guideline on the management of asthma. Thorax 2008; 63: Suppl. 4, iv1-iv121.

2 Bateman ED, Hurd SS, Barnes PJ, et al. Global strategy for asthma management and prevention: GINA executive summary. Eur Respir J 2008; 31: 143-178.

3 Peters SP, Ferguson G, Deniz Y, et al. Uncontrolled asthma: a review of the prevalence, disease burden and options for treatment. Respir Med 2006; 100: 1139-1151.

4 Cazzoletti L, Marcon A, Janson C, et al. Asthma control in Europe: a real-world evaluation based on an international population-based study. J Allergy Clin Immunol 2007; 120: 1360-1367.

5 Williams LK, Pladevall $\mathrm{M}, \mathrm{Xi} \mathrm{H}$, et al. Relationship between adherence to inhaled corticosteroids and poor outcomes among adults with asthma. J Allergy Clin Immunol 2004; 114: 1288-1293.

6 Haughney J, Price D, Kaplan A, et al. Achieving asthma control in practice: understanding the reasons for poor control. Respir Med 2008; 102: 1681-1693.

7 Bender BG, Rand C. Medication non-adherence and asthma treatment cost. Curr Opin Allergy Clin Immunol 2004; 4: 191-195.

8 Bender B, Milgrom H, Apter A. Adherence intervention research: what have we learned and what do we do next? J Allergy Clin Immunol 2003; 112: 489-494.

9 Oberjé EJ, de Kinderen RJ, Evers SM, et al. Cost effectiveness of medication adherence-enhancing interventions: a systematic review of trial-based economic evaluations. Pharmacoeconomics 2013; 31: 1155-1168.

10 Haynes RB, Ackloo E, Sahota N, et al. Interventions for enhancing medication adherence. Cochrane Database Syst Rev 2008; 2: CD000011.

11 Bender BG, Bender SE. Patient-identified barriers to asthma treatment adherence: responses to interviews, focus groups, and questionnaires. Immunol Allergy Clin North Am 2005; 25: 107-130.

12 Bender B, Milgrom H, Rand C. Nonadherence in asthmatic patients: is there a solution to the problem? Ann Allergy Asthma Immunol 1997; 79: 177-185. 
13 Schmier JK, Leidy NK. The complexity of treatment adherence in adults with asthma: challenges and opportunities. J Asthma 1998; 35: 455-472.

14 Spector S. Noncompliance with asthma therapy - are there solutions? J Asthma 2000; 37: 381-388.

15 Weinstein AG. Should patients with persistent severe asthma be monitored for medication adherence? Ann Allergy Asthma Immunol 2005; 94: 251-257.

16 Gillisen A. Patient's adherence in asthma. J Physiol Pharmacol 2007; 58: Suppl. 5, 205-222.

17 Howell G. Nonadherence to medical therapy in asthma: risk factors, barriers, and strategies for improving. J Asthma 2008; 45: 723-729.

18 Otsuki-Clutter M, Sutter M, Ewig J. Promoting adherence to inhaled corticosteroid therapy in patients with asthma. J Clin Outcomes Manage 2011; 18: 177-182.

19 Drotar D, Bonner MS. Influences on adherence to pediatric asthma treatment: a review of correlates and predictors. J Dev Behav Pediatr 2009; 30: 574-582.

20 Charles C, Ninot G, Sultan S. Représentations des patients et observance des traitements par corticostéroïdes inhales dans l'asthme. Revue systématique sur la période 1999-2009 [Patients' illness perceptions and adherence to treatment with inhaled corticosteroids in asthma]. Rev Mal Respir 2011; 28: 626-635.

21 Montori VM, Swiontkowski MF, Cook DJ. Methodologic issues in systematic reviews and meta-analyses. Clin Orthop 2003; 43-54.

22 Moher D, Liberati A, Tetzlaff J, et al. Preferred reporting items for systematic reviews and meta-analyses: the PRISMA statement. PLoS Med 2009; 6: e1000097.

23 Sanderson S, Tatt ID, Higgins JP. Tools for assessing quality and susceptibility to bias in observational studies in epidemiology: a systematic review and annotated bibliography. Int J Epidemiol 2007; 36: 666-676.

24 United Nations Development Programme (UNDP). Human Development Statistical Annex. New York, UNDP, 2011.

25 Von Elm E, Altman DG, Egger M, et al. The Strengthening the Reporting of Observational Studies in Epidemiology (STROBE) Statement: guidelines for reporting observational studies. PLoS Med 2007; 4: e296.

26 Vandenbroucke JP, von Elm E, Altman DG, et al. Strengthening the Reporting of Observational Studies in Epidemiology (STROBE): explanation and elaboration. PLoS Med 2007; 4: e297.

27 Vrijens B, De Geest S, Hughes DA, et al. A new taxonomy for describing and defining adherence to medications. Br J Clin Pharmacol 2012; 73: 691-705.

28 Cohen J. Weighted kappa: nominal scale agreement with provision for scaled disagreement or partial credit. Psychol Bull 1968; 70: 213-220.

29 Altman DG. Practical statistics for medical research. London, Chapman and Hall, 1991.

30 van der Ark LA. Mokken scale analysis in R. I Stat Softw 2007; 20: 1-19.

31 van der Ark LA. New developments in Mokken scale analysis in R. J Stat Softw 2012; 48: 1-27.

32 Tettersell MJ. Asthma patients' knowledge in relation to compliance with drug therapy. J Adv Nurs 1993; 18: 103-113.

33 Bosley CM, Fosbury JA, Cochrane GM. The psychological factors associated with poor compliance with treatment in asthma. Eur Respir J 1995; 8: 899-904.

34 Apter AJ, Reisine ST, Affleck G, et al. Adherence with twice-daily dosing of inhaled steroids. Socioeconomic and health-belief differences. Am J Respir Crit Care Med 1998; 157: 1810-1817.

35 Bennett P, Rowe A, Katz D. Reported adherence with preventive asthma medication: a test of protection motivation theory. Psychol Health Med 1998; 3: 347-354.

36 Chambers CV, Markson L, Diamond JJ, et al. Health beliefs and compliance with inhaled corticosteroids by asthmatic patients in primary care practices. Respir Med 1999; 93: 88-94.

37 Schmaling KB, Afari N, Blume AW. Assessment of psychological factors associated with adherence to medication regimens among adult patients with asthma. J Asthma 2000; 37: 335-343.

38 Horne R, Weinman J. Self-regulation and self-management in asthma: exploring the role of illness perceptions and treatment beliefs in explaining non-adherence to preventer medication. Psychol Health 2002; 17: 17-32.

39 van Schayck CP, Bijl-Hofland ID, Folgering H, et al. Influence of two different inhalation devices on therapy compliance in asthmatic patients. Scand J Prim Health Care 2002; 20: 126-128.

40 Apter AJ, Boston RC, George M, et al. Modifiable barriers to adherence to inhaled steroids among adults with asthma: it's not just black and white. J Allergy Clin Immunol 2003; 111: 1219-1226.

41 Jessop DC, Rutter DR. Adherence to asthma medications: the role of illness representations. Psychol Health 2003; 18: 595-612.

42 Labrecque $\mathrm{M}$, Laurier $\mathrm{C}$, Champagne $\mathrm{F}$, et al. Effect of age on the conformity rate to short-acting $\beta$-agonist use criteria in asthma. J Asthma 2003; 40: 829-835.

43 Nishiyama T, Chrystyn $\mathrm{H}$. The Jones Morbidity Index as an aid for community pharmacists to identify poor asthma control during the dispensing process. Int J Pharm Pract 2003; 11: 41-46.

44 Balkrishnan R, Nelsen LM, Kulkarni AS, et al. Outcomes associated with initiation of different controller therapies in a Medicaid asthmatic population: a retrospective data analysis. J Asthma 2005; 42: 35-40.

45 Lacasse $\mathrm{Y}$, Archibald $\mathrm{H}$, Ernst $\mathrm{P}$, et al. Patterns and determinants of compliance with inhaled steroids in adults with asthma. Can Respir J 2005; 12: 211-217.

46 Stempel DA, Stoloff SW, Carranza Rosenzweig JR, et al. Adherence to asthma controller medication regimens. Respir Med 2005; 99: 1263-1267.

47 Bender BG, Pedan A, Varasteh LT. Adherence and persistence with fluticasone propionate/salmeterol combination therapy. J Allergy Clin Immunol 2006; 118: 899-904.

48 Chatkin JM, Blanco DC, Scaglia N, et al. Impact of a low-cost and simple intervention in enhancing treatment adherence in a brazilian asthma sample. J Asthma 2006; 43: 263-266.

49 Hasegawa T, Suzuki E, Fujimori K, et al. Comparison between compliance of fluticasone propionate diskhaler and of fluticasone propionate diskus in adult bronchial asthma patients. Respiration 2006; 73: 680-684.

50 Marceau C, Lemière C, Berbiche D, et al. Persistence, adherence, and effectiveness of combination therapy among adult patients with asthma. J Allergy Clin Immunol 2006; 118: 574-581.

$51 \mathrm{Ohm}$ R, Aaronson LS. Symptom perception and adherence to asthma controller medications. J Nurs Scholarsh 2006; 38: 292-297. 
52 Tavasoli S, Heidarnazhad H, Kazemnejad A. Factors affecting patients' compliance to metered-dose inhaler drugs in two asthma clinics in Tehran, Iran. Iran J Allergy Asthma Immunol 2006; 5: 187-193.

53 Ulrik CS, Backer V, Søes-Petersen U, et al. The patient's perspective: adherence or non-adherence to asthma controller therapy. J Asthma 2006; 43: 701-704.

54 Williams LK, Joseph CL, Peterson EL, et al. Race-ethnicity, crime, and other factors associated with adherence to inhaled corticosteroids. J Allergy Clin Immunol 2007; 119: 168-175.

55 Williams LK, Joseph CL, Peterson EL, et al. Patients with asthma who do not fill their inhaled corticosteroids: a study of primary nonadherence. J Allergy Clin Immunol 2007; 120: 1153-1159.

56 Breekveldt-Postma NS, Koerselman J, Erkens JA, et al. Treatment with inhaled corticosteroids in asthma is too often discontinued. Pharmacoepidemiol Drug Saf 2008; 17: 411-422.

57 Janson SL, Earnest G, Wong KP, et al. Predictors of asthma medication nonadherence. Heart Lung J Crit Care 2008; 37: 211-218.

58 Martínez-Moragón E, Perpiñá M, Fullana J, et al. Percepción de la disnea y cumplimiento terapéutico en pacientes con asma [Perception of dyspnea and treatment adherence in asthmatic patients]. Arch Bronconeumol 2008; 44: 459-463.

59 McGann EF, Sexton D, Chyun DA. Denial and compliance in adults with asthma. Clin Nurs Res 2008; 17: 151-170.

60 Menckeberg TT, Bouvy ML, Bracke M, et al. Beliefs about medicines predict refill adherence to inhaled corticosteroids. J Psychosom Res 2008; 64: 47-54.

61 Wells K, Pladevall M, Peterson EL, et al. Race-ethnic differences in factors associated with inhaled steroid adherence among adults with asthma. Am J Respir Crit Care Med 2008; 178: 1194-1201.

62 Axelsson M, Emilsson M, Brink E, et al. Personality, adherence, asthma control and health-related quality of life in young adult asthmatics. Respir Med 2009; 103: 1033-1040.

63 Bae Y-J, Kim T-B, Jee Y-K, et al. Severe asthma patients in Korea overestimate their adherence to inhaled corticosteroids. J Asthma 2009; 46: 591-595.

64 Laforest L, El Hasnaoui A, Pribil C, et al. Asthma patients' self-reported behaviours toward inhaled corticosteroids. Respir Med 2009; 103: 1366-1375.

65 Ponieman D, Wisnivesky JP, Leventhal H, et al. Impact of positive and negative beliefs about inhaled corticosteroids on adherence in inner-city asthmatic patients. Ann Allergy Asthma Immunol 2009; 103: 38-42.

66 Friedman HS, Navaratnam P, McLaughlin J. Adherence and asthma control with mometasone furoate versus fluticasone propionate in adolescents and young adults with mild asthma. J Asthma 2010; 47: 994-1000.

67 Takemura M, Kobayashi M, Kimura K, et al. Repeated instruction on inhalation technique improves adherence to the therapeutic regimen in asthma. J Asthma 2010; 47: 202-208.

68 Bolman C, Arwert TG, Völlink T. Adherence to prophylactic asthma medication: habit strength and cognitions. Heart Lung 2011; 40: 63-75.

69 Emilsson M, Berndtsson I, Lötvall J, et al. The influence of personality traits and beliefs about medicines on adherence to asthma treatment. Prim Care Respir J 2011; 20: 141-147.

70 Small M, Anderson P, Vickers A, et al. Importance of inhaler-device satisfaction in asthma treatment: real-world observations of physician-observed compliance and clinical/patient-reported outcomes. Adv Ther 2011; 28: 202-212.

71 Suzuki T, Saito I, Adachi M, et al. Influence of patients' adherence to medication, patient background and physicians' compliance to the guidelines on asthma control. Yakugaku Zasshi 2011; 131: 129-138.

72 Foster JM, Smith L, Bosnic-Anticevich SZ, et al. Identifying patient-specific beliefs and behaviours for conversations about adherence in asthma. Intern Med J 2012; 42: e136-e144.

73 Ahmedani BK, Peterson EL, Wells KE, et al. Asthma medication adherence: the role of God and other health locus of control factors. Ann Allergy Asthma Immunol 2013; 111: 216-220.

74 Axelsson M, Cliffordson C, Lundback B, et al. The function of medication beliefs as mediators between personality traits and adherence behavior in people with asthma. Patient Prefer Adherence 2013; 7: 1101-1109.

75 Price D, Lee AJ, Sims EJ, et al. Characteristics of patients preferring once-daily controller therapy for asthma and COPD: a retrospective cohort study. Prim Care Respir J 2013; 22: 161-168.

76 Price D, Thomas M, Haughney J, et al. Real-life comparison of beclometasone dipropionate as an extrafine- or larger-particle formulation for asthma. Respir Med 2013; 107: 987-1000.

77 Schatz M, Zeiger RS, Yang S-J, et al. Development and preliminary validation of the adult asthma adherence Questionnaire. J Allergy Clin Immunol Pract 2013; 1: 208-288.

78 Wells KE, Peterson EL, Ahmedani BK, et al. Real-world effects of once vs greater daily inhaled corticosteroid dosing on medication adherence. Ann Allergy Asthma Immunol 2013; 111: 216-220.

79 Baddar S, Jayakrishnan B, Al-Rawas OA. Asthma control: importance of compliance and inhaler technique assessments. J Asthma 2014; 51: 429-431.

80 Federman AD, Wolf MS, Sofianou A, et al. Self-management behaviors in older adults with asthma: associations with health literacy. J Am Geriatr Soc 2014; 62: 872-879.

81 Taylor A, Chen L, Smith M. Adherence to inhaled corticosteroids by asthmatic patients: measurement and modelling. J Clin Pharm 2014; 36: 112-119.

82 Van Steenis M, Driesenaar J, Bensing J, et al. Relationship between medication beliefs, self-reported and refill adherence, and symptoms in patients with asthma using inhaled corticosteroids. Patient Prefer Adherence 2014; 8: 83-91.

83 Brooks CM, Richards JM, Kohler CL, et al. Assessing adherence to asthma medication and inhaler regimens: a psychometric analysis of adult self-report scales. Med Care 1994; 32: 298-307.

84 Morisky DE, Green LW, Levine DM. Concurrent and predictive validity of a self-reported measure of medication adherence. Med Care 1986; 24: 67-74.

85 Kardas P, Lewek P, Matyjaszczyk M. Determinants of patient adherence: a review of systematic reviews. Pharm Med Outcomes Res 2013; 4: 91.

86 Karamanidou C, Clatworthy J, Weinman J, et al. A systematic review of the prevalence and determinants of nonadherence to phosphate binding medication in patients with end-stage renal disease. BMC Nephrol 2008; 9: 2 .

87 Mills EJ, Nachega JB, Bangsberg DR, et al. Adherence to HAART: a systematic review of developed and developing nation patient-reported barriers and facilitators. PLoS Med 2006; 3: e438. 
88 Vermeire E, Hearnshaw H, Van Royen $\mathrm{P}$, et al. Patient adherence to treatment: three decades of research. A comprehensive review. J Clin Pharm Ther 2001; 26: 331-342.

89 World Health Organization. Innovative Care for Chronic Conditions: Building Blocks for Action. Global report. Geneva, WHO, 2002.

90 De Bruin M, Viechtbauer W, Hospers HJ, et al. Standard care quality determines treatment outcomes in control groups of HAART-adherence intervention studies: implications for the interpretation and comparison of intervention effects. Health Psychol 2009; 28: 668-674.

91 de Bruin M, Viechtbauer W, Schaalma HP, et al. Standard care impact on effects of highly active antiretroviral therapy adherence interventions: a meta-analysis of randomized controlled trials. Arch Intern Med 2010; 170: 240-250.

92 Bartholomew LK, Parcel GS, Kok G, et al. Planning Health Promotion Programs: an Intervention Mapping Approach. 3rd edn. San Francisco, Jossey-Bass, 2011.

93 Gwadry-Sridhar FH, Manias E, Zhang Y, et al. A framework for planning and critiquing medication compliance and persistence research using prospective study designs. Clin Ther 2009; 31: 421-435.

94 Martin LR, Haskard-Zolnierek KB, DiMatteo MR. Health Behavior Change and Treatment Adherence: Evidence-Based Guidelines for Improving Healthcare. New York, Oxford University Press, 2010.

95 World Health Organization. Adherence to Long-Term Therapies: Evidence for Action. Geneva, WHO, 2003. 